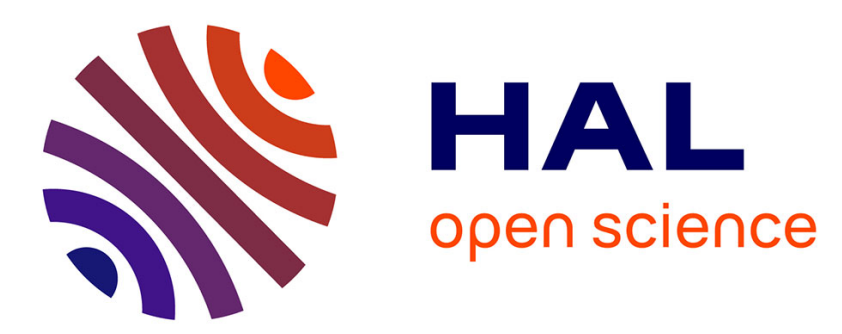

\title{
Propagation of acoustic waves through saturated porous media
}

J.-F. Thovert, Xiang-Yu Li, I. Malinouskaya, Valeri V. Mourzenko, P. Adler

\section{To cite this version:}

J.-F. Thovert, Xiang-Yu Li, I. Malinouskaya, Valeri V. Mourzenko, P. Adler. Propagation of acoustic waves through saturated porous media. Physical Review E , 2020, 102 (2), pp.023001(15). 10.1103/PhysRevE.102.023001 . hal-02986695

\section{HAL Id: hal-02986695 \\ https://hal.science/hal-02986695}

Submitted on 10 Nov 2020

HAL is a multi-disciplinary open access archive for the deposit and dissemination of scientific research documents, whether they are published or not. The documents may come from teaching and research institutions in France or abroad, or from public or private research centers.
L'archive ouverte pluridisciplinaire HAL, est destinée au dépôt et à la diffusion de documents scientifiques de niveau recherche, publiés ou non, émanant des établissements d'enseignement et de recherche français ou étrangers, des laboratoires publics ou privés. 


\title{
Propagation of acoustic waves through saturated porous media
}

\author{
J.-F. Thovert $\odot,{ }^{1}$ Xiang-Yu Li, ${ }^{1, *}$ I. Malinouskaya $\odot,{ }^{1, \dagger}{ }^{\dagger}$ V. V. Mourzenko, ${ }^{1}$ and P. M. Adler $\odot^{2, *}$ \\ ${ }^{1}$ Institut P', CNRS, Université de Poitiers, ISAE-ENSMA, 11 bd Marie et Pierre Curie, TSA 41123, 86073 Poiters Cedex 9, France \\ ${ }^{2}$ Metis, Sorbonne Université, 75252 Paris Cedex 05, France
}

(Received 22 May 2020; accepted 7 July 2020; published 3 August 2020)

\begin{abstract}
The homogenization approach to wave propagation through saturated porous media is extended in order to include the compressibility of the interstitial fluid and the existence of several connected pore components which may or not percolate. The necessary theoretical developments are summarized and the Christoffel equation whose solutions provide the wave velocities is presented. Some analytical developments are proposed for isotropic media. Finally, a systematic application to a synthetic porous medium illustrates the methodology and its results.
\end{abstract}

DOI: 10.1103/PhysRevE.102.023001

\section{INTRODUCTION}

Since the pioneering contribution of Biot [1], wave propagation through saturated porous media has generated a lot of theoretical and practical interest. In underground media, in most cases, waves propagate through a heterogeneous medium which contains at least one fluid and often two, such as oil and/or water. The presence of these fluids influences the wave celerities.

The homogenization technique was used much later to derive the macroscopic mechanical behavior of a porous medium saturated with an incompressible fluid [2,3] or with a compressible one [4]. These first analyses extended in [5] and [6] were applied in [7] to numerically calculate the wave velocities in a porous medium consisting of a single connected void filled with an incompressible fluid. A general literature survey is also found in [7], which can be completed as follows.

Reference [8] considered isotropic multiple-porosity systems, but the authors started from the Biot equations, in contrast with the previous references, which started at the local scale. Reference [9] was a study of acoustical waves in a porous medium composed of two independent connected pore components, each one filled with an incompressible fluid.

In [10], the effect of pore fluid viscosity on effective elastic properties using digitized rocks was studied; a significant velocity dispersion in wave propagation simulations was obtained by varying the pore fluid viscosity; the study was not performed in the framework of homogenization theory. The same group [11] studied wave propagation through metallic foams by direct simulation.

Numerical homogenization techniques for analyzing transport properties in real foam samples, mostly open cell, were applied in [12] to understand long-wavelength acoustics of

\footnotetext{
*Present address: Southwest Jiao-tong University, Chengdu, Sichuan 610031, China.

${ }^{\dagger}$ Present address: IFP Energies Nouvelles, 92852 Rueil-

Malmaison, France.

ॠpierre.adler@upmc.fr
}

rigid-frame air-saturated porous media on the basis of microstructural parameters; experimental characterization of the porosity and permeability of real foam samples were used to provide the scaling of a polyhedral unit cell.

As will be shown, the dynamic permeability is necessary in order to determine wave velocities through porous media; a fast Fourier transform-based method for computing the dynamic permeability of periodic porous media was presented in [13], which is efficient and compares well with existing results. The same group worked on the elastic and acoustic properties of a regular solid foam in the framework of homogenization theory [14].

Finally, in [15], multiscale modeling allowed the determination of the effective speeds and damping of acoustic waves propagating in fibrous media, which brought up a discussion of the correlation between the speed, penetration range, and attenuation of sound waves.

The major objective of the present study is to extend [7] in two important ways. The first is to include the compressibility of the fluid, a feature which was not included in the phenomenology of [7]. The second is to cope with porous media whose pore space may have several connected components. Practically speaking, this feature is always present in real porous media where closed porosity exists. Note that this is different from the multiple porosity in [8], which represents a mesoscopically heterogeneous medium with a mixture of two porous media, each containing a singly connected pore system.

This paper is organized as follows. Section II provides the general physical background, the basic equations which govern wave propagation in saturated porous media, and the basic analytical tool, which is the double-scale expansion technique.

Most of the theoretical analysis is contained in Sec. III. It should be emphasized that we tried to keep duplication of [7] to a minimum. However, for the sake of clarity, it was not always possible to avoid it completely. The microscopic equations of the various orders are presented first. Then they are analyzed and the macroscopic coefficients to be used in the wave velocity computations are defined. Finally, the 
macroscopic equations which govern wave propagation on the macroscopic scale are provided.

This set of theoretical results is discussed in Sec. IV. First, they are compared to the ones given in [7]. The influence of the fluid compressibility and of several pore components is detailed. Then the macroscopic equations are reformulated in a compact way which makes apparent their correspondence with the simpler system used in [7]. The meaning and the properties of the macroscopic coefficients which often are tensors are recalled, as well as the associated closure problem from which they are derived.

The characteristics of plane waves are analyzed in Sec. V and this yields the classical Christoffel equation. Some theoretical consequences for macroscopic isotropic media are developed. A detailed application is presented in Sec. VI for a medium which contains open and closed pores. Finally, in Sec. VII, some concluding remarks terminate the paper.

\section{GENERAL}

\section{A. Physical background}

Wave propagation is analyzed through fluid-saturated porous media. The porous matrix is an elastic heterogeneous material. The size $l$ of the heterogeneities (scale of the geometrical microstructure or of property variations) is supposed to be much smaller than the overall dimension Łof the domain. We assume that the material is statistically homogeneous on some intermediate scale between $l$ and Łand therefore it can be regarded as spatially periodic, i.e., it is made of identical unit cells $\Omega$ of size $L$.

Despite the two very significant extensions to compressible fluid and medium with multiple pores, the analysis is similar to that in [7] (with a detailed approach in [16]) and is conducted in the framework of the homogenization theory, with the double-scale expansion technique of [5] and additional elements regarding the compressibility effects taken from [17]. More precisely, the domain $\Omega$ is composed of a totally connected region $\Omega_{s}$, filled with solid material, and a pore space $\Omega_{f}$, which can involve several independent connected components $\Omega_{f}^{i}, i=1,2, \ldots, N$. Some of these components (generally at most one) can be percolating, but this is not a requirement. Hence

$$
\Omega=\Omega_{s} \cup \Omega_{f}, \quad \Omega_{f}=\bigcup_{i=1}^{N} \Omega_{f}^{i} .
$$

Throughout this paper, the superscript $i$ is used to refer to the pore components and the quantities associated with them (volume fraction $\epsilon^{i}$, fluid pore pressure $P^{i}$, etc).

All the pores are assumed to be filled with an identical compressible fluid. With respect to some reference state $\left(P_{0}\right.$, $\left.\rho_{0}\right)$, the pressure and density variations are related by

$$
P-P_{0}=a_{0}^{2}\left(\rho_{f}-\rho_{0}\right),
$$

where $a_{0}^{2}$ is the speed of sound in the fluid, related to the compressibility coefficient $c_{f}$ by $a_{0}=1 / \sqrt{\rho_{0} c_{f}}$.

We analyze here disturbances from the reference state (which is generally prestrained, such as in situ conditions) caused by acoustic waves. Hence, all the deformations and stresses are measured relative to the reference state, and $P_{0}$ is omitted in the formulation.
We consider the propagation in this medium of a harmonic wave of pulsation $\omega$ and wavelength $\lambda$ intermediate between $l$ and $€$. In the following, the length $\mathcal{L}$ is used as a characteristic macroscopic scale,

$$
\mathcal{L}=\lambda / 2 \pi \text {. }
$$

Quantities such as the solid displacement $\boldsymbol{u}_{s}$ and the fluid velocity $\boldsymbol{V}$ are assumed to be of the form $\widehat{\boldsymbol{A}} e^{i \omega t}$.

The separation between the microscale $l$ and the macroscale $\mathcal{L}$ allows us to use a homogenization procedure in order to derive the macroscopic characteristics of the heterogeneous medium by solving a series of local problems. This method is based on a double-scale expansion of the governing equations in terms of slow- and fast-varying space variables which are related by the small parameter $\eta$,

$$
\eta=l / \mathcal{L} \ll 1 .
$$

\section{B. Basic equations}

All the quantities relative to the solid and fluid phases are denoted by the subscripts $s$ and $f$, respectively. The wave propagation in saturated porous media is governed on the microscopic level in the solid matrix $\Omega_{s}$ by the elastic equation

$$
\nabla \cdot \boldsymbol{\sigma}_{s}=-\rho_{s} \omega^{2} \boldsymbol{u}_{s} \quad \text { in } \Omega_{s},
$$

where $\rho_{s}$ is the density of the solid material; $\sigma_{s}$ is the stress tensor related to the strain tensor $\boldsymbol{e}_{s}$ by the solid stiffness tensor $\boldsymbol{C}$,

$$
\sigma_{s}=C: e_{s}
$$

and the displacement $\boldsymbol{u}_{s}$ is of the form

$$
\boldsymbol{u}_{s}=\widehat{\boldsymbol{u}}_{s} e^{i \omega t}
$$

The fluid displacements $\boldsymbol{u}_{f}$ are also of the harmonic form, (7). Hence, the fluid velocity $\boldsymbol{V}$ is given by

$$
\boldsymbol{V}=\frac{\partial \boldsymbol{u}_{f}}{\partial t}=i \omega \boldsymbol{u}_{f}
$$

The Navier-Stokes equations for the fluid motion in $\Omega_{f}$ can be written

$$
\begin{aligned}
& \nabla \cdot \boldsymbol{\sigma}_{f}=\rho_{f}\left[\frac{\partial \boldsymbol{V}}{\partial t}+\boldsymbol{V} \cdot \nabla \boldsymbol{V}\right], \\
& \frac{\partial \rho_{f}}{\partial t}+\nabla \cdot\left(\rho_{f} \boldsymbol{V}\right)=0,
\end{aligned}
$$

where $\sigma_{f}$ is the fluid stress tensor given by

$$
\begin{aligned}
\boldsymbol{\sigma}_{f} & =-P \boldsymbol{I}+2 \mu \mathcal{D}(\boldsymbol{V}), \\
\mathcal{D}(\boldsymbol{V}) & =\frac{1}{2}\left(\nabla \boldsymbol{V}+{ }^{t} \nabla \boldsymbol{V}\right)-\frac{2}{3}(\nabla \cdot \boldsymbol{V}) \boldsymbol{I},
\end{aligned}
$$

where $\mu$ is the dynamic viscosity. Following [17], it is assumed that the acoustic wave amplitude is low enough so that the disturbances with respect to the rest state $\left(\rho_{f}=\rho_{0}, P=0\right.$, $\boldsymbol{V}=0$ ) are of a relative magnitude $\xi \ll \eta$, i.e.,

$$
\frac{V}{\omega l}, \quad \frac{\rho_{f}-\rho_{0}}{\rho_{0}} \sim O(\xi) \ll \eta .
$$

Therefore, by ignoring $o\left(\xi^{2}\right)$ terms and by using (7) and (8), the Navier-Stokes equations can be linearized into

$$
i \rho_{0} \omega \boldsymbol{V}=\nabla \cdot \sigma_{f}, \quad i \omega\left(\rho_{f}-\rho_{0}\right)+\rho_{0} \nabla \cdot \boldsymbol{V}=0 .
$$


Continuity of displacements and normal stresses at the solidfluid interfaces $\Gamma^{i}=\Omega_{s} \cap \Omega_{f}^{i}$ implies that

$$
\boldsymbol{u}_{s}=\boldsymbol{u}_{f}^{i} \quad \text { on } \quad \Gamma^{i}, \quad\left(\boldsymbol{\sigma}_{s}-\boldsymbol{\sigma}_{f}^{i}\right) \cdot \boldsymbol{n}^{i}=0 \quad \text { on } \quad \Gamma^{i},
$$

where $\boldsymbol{n}^{i}$ is the unit normal to $\Gamma^{i}$, oriented from solid to pore.

There are three different modes of wave propagation in such media, according to the range of frequency. They are discussed in [7] and this discussion is not repeated here. We focus here on the biphasic regime only, which corresponds to the low-frequency range studied in [1]. Only the conditions and their physical meaning are recalled at the beginning of Sec. III.

\section{Double-scale expansion in periodic heterogeneous media}

Let us assume that the medium is spatially periodic as described in Sec. II. Moreover, the lengths $l, L, \mathcal{L}$, and $\lambda$ verify

$$
l \leqslant L \ll \mathcal{L}=\lambda / 2 \pi \text {. }
$$

In order to distinguish between the macroscopic and the microscopic scales, two spatial variables, $\boldsymbol{x}$ and $\boldsymbol{y}$, are introduced (see [5] and [7]). $\boldsymbol{x}$ is macroscopic, i.e., of order $\mathcal{L}$, while $\boldsymbol{y}$ is microscopic, i.e., of order $l$. Therefore,

$$
\boldsymbol{y}=\eta^{-1} \boldsymbol{x} .
$$

Consequently, the spatial gradient operator becomes

$$
\nabla=\nabla_{x}+\eta^{-1} \nabla_{y}
$$

where $\nabla_{x}$ and $\nabla_{y}$ are differential operators with respect to the $\boldsymbol{x}$ and $\boldsymbol{y}$ variables, respectively. Any function of space can be represented as a function of these two spatial variables $\boldsymbol{f}(\boldsymbol{x}, \boldsymbol{y})$. Because of the spatial periodicity of the medium, $f$ is periodic in $\boldsymbol{y}$; moreover, $\boldsymbol{f}$ can be expanded as a series in terms of the small parameter $\eta$,

$$
\boldsymbol{f}(\boldsymbol{x}, \boldsymbol{y})=\sum \eta^{j} \boldsymbol{f}^{(j)}(\boldsymbol{x}, \boldsymbol{y})
$$

where $\boldsymbol{f}^{(j)}(\boldsymbol{x}, \boldsymbol{y})$ is also a periodic function of $\boldsymbol{y}$. This expansion is applied to the displacements $\boldsymbol{u}_{s}$ and $\boldsymbol{u}_{f}$, the velocity $\boldsymbol{V}$, the fluid stress tensor $\sigma_{f}$, the fluid density $\rho_{f}$, and the pressure $P$ with an obvious notation; the superscript $(j)$ denotes the order.

Substitution of the expansion of $\boldsymbol{u}_{s}$, (17), into Eq. (5) yields the same expansions for the deformation operator $\boldsymbol{e}_{s}$ and the stress tensor $\sigma_{s}$. Then a series of equations for the solid phase for each power of $\eta$ is obtained identical to Eq. (14) in [7].

Substitution of the expansions, (17), for $\boldsymbol{V}$ and $\boldsymbol{\sigma}_{f}$ into Eq. (12) yields a series of equations for the fluid phase similar to that for the solid one, for each power of $\eta$ :

$$
\begin{array}{ll}
\eta^{-2}: & \nabla_{y} \cdot \boldsymbol{\sigma}_{f}^{(-1)}=0, \\
\eta^{-1}: & \nabla_{y} \cdot \boldsymbol{\sigma}_{f}^{(0)}+\nabla_{x} \cdot \boldsymbol{\sigma}_{f}^{(-1)}=0, \\
\eta^{0}: & \nabla_{y} \cdot \boldsymbol{\sigma}_{f}^{(1)}+\nabla_{x} \cdot \boldsymbol{\sigma}_{f}^{(0)}=\rho_{f} i \omega \boldsymbol{V}^{(0)}, \\
\vdots & \nabla_{y} \cdot \boldsymbol{\sigma}_{f}^{(j+1)}+\nabla_{x} \cdot \boldsymbol{\sigma}_{f}^{(j)}=\rho_{f} i \omega \boldsymbol{V}^{(j)}, \\
\eta^{j}: & \nabla_{y} \cdot \boldsymbol{V}^{(0)}=0, \\
{\left[\nabla_{y} \cdot \boldsymbol{V}^{(j+1)}+\nabla_{x} \cdot \boldsymbol{V}^{(j)}\right] \rho_{0}=-i \omega \rho_{f}^{(j)},} \\
j=0,1,2,3, \ldots
\end{array}
$$

The rate of deformation $\mathcal{D}$ can be derived from (17) for $\boldsymbol{V}$ as

$$
\mathcal{D}(\boldsymbol{V})=\eta^{-1} \mathcal{D}_{\boldsymbol{y}}\left(\boldsymbol{V}^{(0)}\right)+\sum_{j=0}^{\infty} \eta^{j}\left[\mathcal{D}_{\boldsymbol{x}}\left(\boldsymbol{V}^{(j)}\right)+\mathcal{D}_{\boldsymbol{y}}\left(\boldsymbol{V}^{(j+1)}\right)\right]
$$

where $\mathcal{D}_{\boldsymbol{x}}$ and $\mathcal{D}_{\boldsymbol{y}}$ denote the operator $\mathcal{D}$ applied to the $\boldsymbol{x}$ and $\boldsymbol{y}$ variables, respectively.

Note that Eqs. (18) and (19) have to be applied in each of the pore components $\Omega_{f}^{i}$, which yields $N$ independent flow problems. For each of them, the following conditions for each power of $\eta$ at the solid-fluid interface are obtained:

$\boldsymbol{u}_{s}^{(j)}=\boldsymbol{u}_{f}^{(j)} \quad$ on $\quad \Gamma^{i}, \quad\left(\boldsymbol{\sigma}_{s}^{(j)}-\boldsymbol{\sigma}_{f}^{(j)}\right) \cdot \boldsymbol{n}=0 \quad$ on $\quad \Gamma^{i}$.

At this point, the full procedure demands that the equations are made dimensionless and that the orders of magnitude of the various terms are compared, taking into account the targeted range of frequency quantified by the transient Reynolds number $R_{T}$ :

$$
R_{T}=\frac{\omega \rho_{f} l^{2}}{\mu} .
$$

For the sake of brevity, this step was skipped, as in [5] and [7]. We focus in the following on the low-frequency range $R_{T}=$ $O(1)$, which gives rise to the biphasic regime described by Biot equations [1].

\section{THE BIPHASIC MACROSCOPIC BEHAVIOR OF A SATURATED POROUS MEDIUM: ANALYSIS}

Wave propagation is in the biphasic macroscopic mode if the following two conditions are satisfied:

$$
R_{T}=O(1), \quad \frac{\mu \omega}{\left\|C_{\{4\}}\right\|}=O\left(\eta^{2}\right)
$$

The physical meaning of these conditions is obvious. The first one implies that the partial time derivative in the NavierStokes equation is of the same order of magnitude as the viscous terms. The second one implies that the viscous stress is of the same order of magnitude as the elastic stress at the solid-liquid interface.

Because of the interdependence of the solid and fluid equations, the wave celerity depends on the effective elastic properties of the matrix, on the permeability, and on the mechanical reaction of the solid on the fluid pressure [5], i.e., on the global characteristics of both phases, thus, the name biphasic macroscopic behavior. Since in this case the relative fluid or solid motion is not 0 , this regime can also be called the drained regime.

Since the contrast between mechanical properties of the solid and fluid is of order $O\left(\eta^{2}\right)$, the fluid stress tensor $\sigma_{f}$ takes the form

$$
\boldsymbol{\sigma}_{f}=-\boldsymbol{I} P+\eta^{2} 2 \mu \mathcal{D}(\boldsymbol{V})
$$

Note that in view of Eq. (10b), an additional dilational viscous term could be expected in Eq. (24). The analysis shows that it does not actually contribute to the considered order. 
Substitution of (17) twice and (20) into (24) yields the expansion of the stress tensor $\sigma_{f},(17)$, as

$$
\begin{aligned}
\boldsymbol{\sigma}_{f}^{(-1)} & =0, \\
\boldsymbol{\sigma}_{f}^{(0)} & =-\boldsymbol{I} P^{(0)}, \\
\boldsymbol{\sigma}_{f}^{(1)} & =-\boldsymbol{I} P^{(1)}+2 \mu \mathcal{D}_{\boldsymbol{y}}\left(\boldsymbol{V}^{(0)}\right),
\end{aligned}
$$

$$
\boldsymbol{\sigma}_{f}^{(j)}=-\boldsymbol{I} \boldsymbol{P}^{(j)}+2 \mu\left(\mathcal{D}_{\boldsymbol{x}}\left(\boldsymbol{V}^{(j-2)}\right)+\mathcal{D}_{\boldsymbol{y}}\left(\boldsymbol{V}^{(j-1)}\right)\right) \quad(j>1) .
$$

Of course, the equations apply independently in each of the pore components $\Omega_{f}^{i}$.

\section{A. The microscopic equations of the various orders}

When expansion (17) for $\sigma_{f}$ and definition (25) are taken into account, the equations and boundary conditions for each power of the small parameter $\eta$ can be derived,

$$
\begin{aligned}
& \eta^{-2}: \nabla_{y} \boldsymbol{C}_{\{4\}}: \boldsymbol{e}\left(\boldsymbol{u}_{s}^{(0)}\right)=0 \quad \text { in } \quad \Omega_{s} \\
& \left\{\boldsymbol{C}_{\{4\}}: \boldsymbol{e}\left(\boldsymbol{u}_{s}^{(0)}\right)\right\} \cdot \boldsymbol{n}^{i}=0 \quad \text { on } \quad \Gamma^{i}, \quad i=1, \ldots, N, \\
& \eta^{-1}: \nabla_{y} \cdot \boldsymbol{C}_{\{4\}}:\left[\boldsymbol{e}\left(\boldsymbol{u}_{s}^{(1)}\right)+\boldsymbol{E}\left(\boldsymbol{u}_{s}^{(0)}\right)\right]+\nabla_{x} \cdot \boldsymbol{C}_{\{4\}}: \boldsymbol{e}\left(\boldsymbol{u}_{s}^{(0)}\right)=0 \quad \text { in } \quad \Omega_{s}, \\
& -\nabla_{y} P^{i(0)}=0 \quad \text { in } \quad \Omega_{f}^{i}, \quad i=1, \ldots, N \text {, } \\
& \left\{\boldsymbol{C}_{\{4\}}:\left[\boldsymbol{e}\left(\boldsymbol{u}_{s}^{(1)}\right)+\boldsymbol{E}\left(\boldsymbol{u}_{s}^{(0)}\right)\right]+\boldsymbol{I} P^{i(0)}\right\} \cdot \boldsymbol{n}^{i}=0 \quad \text { on } \quad \Gamma^{i}, \quad i=1, \ldots, N, \\
& \eta^{0}: \nabla_{y} \cdot \boldsymbol{C}_{\{4\}}:\left[\boldsymbol{e}\left(\boldsymbol{u}_{s}^{(2)}\right)+\boldsymbol{E}\left(\boldsymbol{u}_{s}^{(1)}\right)\right]+\nabla_{x} \cdot \boldsymbol{C}_{\{4\}}:\left[\boldsymbol{e}\left(\boldsymbol{u}_{s}^{(1)}\right)+\boldsymbol{E}\left(\boldsymbol{u}_{s}^{(0)}\right)\right]=-\rho_{s} \omega^{2} \boldsymbol{u}_{s}^{(0)} \quad \text { in } \quad \Omega_{s}, \\
& -\nabla_{y} P^{i(1)}+\nabla_{y} \cdot\left[2 \mu \mathcal{D}_{y}\left(\boldsymbol{V}^{i(0)}\right)\right]-\nabla_{x} P^{i(0)}=i \rho_{0} \omega \boldsymbol{V}^{i(0)} \quad \text { in } \quad \Omega_{f}^{i}, \quad i=1, \ldots, N, \\
& \nabla_{y} \cdot \boldsymbol{V}^{i(0)}=0 \quad \text { in } \quad \Omega_{f}^{i}, \quad i=1, \ldots, N, \\
& \boldsymbol{u}_{s}^{(0)}=\boldsymbol{u}_{f}^{(0)} \quad \text { on } \quad \Gamma^{i}, \quad i=1, \ldots, N, \\
& \left\{\boldsymbol{C}_{\{4\}}:\left[\boldsymbol{e}\left(\boldsymbol{u}_{s}^{(2)}\right)+\boldsymbol{E}\left(\boldsymbol{u}_{s}^{(1)}\right)\right]+\boldsymbol{I} P^{i(1)}-2 \mu \mathcal{D}_{y}\left(\boldsymbol{V}^{i(0)}\right)\right\} \cdot \boldsymbol{n}^{i}=0 \quad \text { on } \quad \Gamma^{i}, \quad i=1, \ldots, N,
\end{aligned}
$$

where $\boldsymbol{n}^{i}$ is the unit normal to $\Gamma^{i} . \boldsymbol{E}$ and $\boldsymbol{e}$ are the macroscopic and microscopic deformations, respectively. Note that the dilational viscous stress vanishes at order $\eta^{0}$, because $\nabla_{y} \cdot \boldsymbol{V}^{(0)}=0$.

These microscopic equations need to be solved first in order to get the variations with the local variable $\boldsymbol{y}$. Then the macroscopic equations which must be verified by plane waves, for instance, are obtained at the order $O\left(\eta^{0}\right)$ and integration over the unit cell; these macroscopic equations contain quantities which can be derived from the solutions of the microscopic equations. This enables us to obtain the global wave characteristics, such as the celerity and the attenuation.

The technique is almost identical to the one detailed in [16], differing by only a few points. Therefore, the following is limited to a presentation of the microscopic equations and of the quantities which appear in the macroscopic equations and in the macroscopic equations.

\section{B. The microscopic equations and their solutions}

The microscopic equation for the solid phase corresponds to the order $O\left(\eta^{-1}\right)$, i.e., to (26b). It is used to derive several macroscopic quantities. The first is the macroscopic (or, equivalently, the effective) stiffness tensor $\boldsymbol{D}_{\{4\}}^{(0)}$ of the dry medium. It can be obtained by solving the time-independent elasticity equation

$$
\begin{aligned}
& \nabla_{y} \cdot \boldsymbol{C}_{\{4\}}:\left[\boldsymbol{e}\left(\boldsymbol{u}_{s}^{(1)}\right)+\boldsymbol{E}\left(\boldsymbol{U}_{s}^{(0)}\right)\right]=0 \text { in } \Omega_{s}, \\
& \left\{\boldsymbol{C}_{\{4\}}:\left[\boldsymbol{e}\left(\boldsymbol{u}_{s}^{(1)}\right)+\boldsymbol{E}\left(\boldsymbol{U}_{s}^{(0)}\right)\right]\right\} \cdot \boldsymbol{n}^{i}=0 \text { on } \Gamma^{i}, \\
& i=1, \ldots, N,
\end{aligned}
$$

where $\boldsymbol{E}\left(\boldsymbol{U}_{s}^{(0)}\right)$ is the imposed macroscopic strain, and $\boldsymbol{e}\left(\boldsymbol{u}_{s}{ }^{(1)}\right)$ the local strain. The macroscopic stiffness tensor $\boldsymbol{D}_{\{4\}}^{(0)}$ is derived by integrating the strain over the unit cell,

$$
\left.\boldsymbol{D}_{\{4\}}^{(0)}: \boldsymbol{E}\left(\boldsymbol{U}_{s}^{(0)}\right)=\left\langle\boldsymbol{C}_{\{4\}}:\left[\boldsymbol{e}\left(\boldsymbol{u}_{s}^{(1)}\right)+\boldsymbol{E}\left(\boldsymbol{U}_{s}^{(0)}\right)\right]\right\}\right\rangle,
$$

where $\langle\circ\rangle$ is the average operator over the unit cell, which is defined as

$$
\langle\circ\rangle=\frac{1}{\boldsymbol{\Omega}} \int_{\boldsymbol{\Omega}} \circ d \boldsymbol{\Omega} .
$$

In [7], one had to determine the displacement field in the solid when a unit pressure was imposed on the unique pore. The tensor $\alpha$ and the scalar $\beta$ characterized this field. Here, one has to perform these calculations in every connected component of the pores. This can be generalized for several pores as follows. The vector $\boldsymbol{q}^{i}$ is the particular solution of the local elasticity equation with $P^{i(0)}=1, P^{j(0)}=0$ for $i \neq j$ and $\boldsymbol{E}\left(\boldsymbol{U}_{s}^{(0)}\right)=0$. In other words, it represents the displacement field in the solid when a unit pressure is imposed on the interstitial fluid in $\Omega_{f}^{i}$ only. Then the equation to determine 
$\boldsymbol{q}^{i}$ can be written as

$$
\begin{aligned}
& \nabla_{y} \cdot\left[\boldsymbol{C}_{\{4\}}: \boldsymbol{e}\left(\boldsymbol{q}^{i}\right)\right]=0 \text { in } \Omega_{s}, \\
& \left\{\boldsymbol{C}_{\{4\}}: \boldsymbol{e}\left(\boldsymbol{q}^{i}\right)\right\} \cdot \boldsymbol{n}^{i}=-\boldsymbol{n}^{i} \text { on } \Gamma^{i}, \\
& \left\{\boldsymbol{C}_{\{4\}}: \boldsymbol{e}\left(\boldsymbol{q}^{j}\right)\right\} \cdot \boldsymbol{n}^{i}=0 \text { on } \Gamma^{j}, \quad j \neq i .
\end{aligned}
$$

Let us denote

$$
\begin{gathered}
\boldsymbol{\alpha}^{i}=-\epsilon^{i} \boldsymbol{I}+\left\langle\boldsymbol{C}_{\{4\}}: \boldsymbol{e}\left(\boldsymbol{q}^{i}\right)\right\rangle, \\
B^{i j}=\frac{1}{\Omega} \int_{\Gamma^{i}} \boldsymbol{n}^{i} \cdot \boldsymbol{q}^{j} d s,
\end{gathered}
$$

where $\epsilon^{i}$ is the volume fraction of $\Omega_{f}^{i}$.

At order $O\left(\eta^{0}\right)$, the Navier-Stokes equation is written in each connected pore component in terms of the fluid velocity relative to the solid $\boldsymbol{W}^{i}$,

$$
\boldsymbol{W}^{i}=\boldsymbol{u}_{f}^{i(0)}-\boldsymbol{U}_{s}^{(0)} .
$$

The vector $\boldsymbol{W}^{i}$ is an $\Omega$-periodic function. The displacement $\boldsymbol{u}_{f}^{i(0)}$ is different from the macroscopic displacement in the solid $\boldsymbol{U}_{s}^{(0)}$, i.e., the vector $\boldsymbol{W}^{i}$ is not 0 and it corresponds to the drained regime. Let us denote its velocity

$$
\mathcal{W}^{i}=i \omega \boldsymbol{W}^{i} \text {. }
$$

It can be shown that $\boldsymbol{W}^{i}$ verifies in pore $i$

$$
\begin{aligned}
& \mu \Delta_{y} \mathcal{W}^{i}-i \rho_{0} \omega \mathcal{W}^{i}-\nabla_{y} P^{i(1)}=\nabla_{x} P^{i(0)}-\rho_{f} \omega^{2} \boldsymbol{U}_{s}^{(0)} \text { in } \Omega_{f}^{i}, \\
& \nabla_{y} \cdot \mathcal{W}^{i}=0 \text { in } \Omega_{f}^{i}, \\
& \mathcal{W}^{i}=0 \text { on } \Gamma^{i},
\end{aligned}
$$

where $P^{i(0)}$ is the pressure inside the pore. The driving force is related to the right-hand side of the dynamic equation

$$
\boldsymbol{G}^{i}=-\nabla_{x} P^{i(0)}+\rho_{0} \omega^{2} \boldsymbol{U}_{s}^{(0)} .
$$

Because of the linearity of the system, (33), $\mathcal{W}^{i}$ can be shown to be linearly related to the driving force

$$
\mathcal{W}^{i}=\frac{1}{\mu} \boldsymbol{k}^{i} \cdot \boldsymbol{G}^{i},
$$

where $\boldsymbol{k}^{i}$ is a complex $\omega$-dependent tensor. Then integration of Eq. (35) over $\Omega_{f}^{i}$ yields the dynamic Darcy law

$$
i \omega\left\langle\boldsymbol{W}^{i}\right\rangle=\frac{1}{\mu} \boldsymbol{K}^{i} \cdot\left(-\nabla_{x} P^{i(0)}+\rho_{0} \omega^{2} \boldsymbol{U}_{s}^{(0)}\right),
$$

where $\boldsymbol{K}^{i}$ is the dynamic permeability tensor for the pore component $\Omega_{f}^{i}$, which takes complex values and depends on the frequency

$$
\boldsymbol{K}^{i}=\left\langle\boldsymbol{k}^{i}\right\rangle
$$

\section{Macroscopic equations}

The set of equations of order $O\left(\eta^{0}\right)$ for the solid and the fluid phases can be written as

$$
\begin{aligned}
\nabla_{y} \cdot \boldsymbol{\sigma}_{s}^{(1)}+\nabla_{x} \cdot \boldsymbol{\sigma}_{s}^{(0)} & =-\rho_{s} \omega^{2} \boldsymbol{U}_{s}^{(0)} \quad \text { in } \quad \Omega_{s}, \\
\nabla_{y} \cdot \boldsymbol{\sigma}_{f}^{(1)}+\nabla_{x} \cdot \boldsymbol{\sigma}_{f}^{i(0)} & =-\rho_{0} \omega^{2} \boldsymbol{u}^{i(0)_{f}} \text { in } \Omega_{f}^{i}, \quad i=1, \ldots, N, \\
\left(\boldsymbol{\sigma}_{s}^{(1)}-\boldsymbol{\sigma}_{f}^{(1)}\right) \cdot \boldsymbol{n}^{i} & =0 \quad \text { on } \quad \Gamma^{i}, \quad i=1, \ldots, N .
\end{aligned}
$$

Then these equations are integrated over the unit cell and the various tensors issued from the microscopic equations in Sec. III B can be used. Again, the derivation closely follows the one detailed in [16], except for the fact that an equation has to be written for each pore $i$. The macroscopic description in terms of the solid displacement $\boldsymbol{U}_{s}^{(0)}$, the pressures $P^{i(0)}$, and the fluid displacements $\boldsymbol{W}^{i(0)}$ in the pores, relative to the solid, involves the elasticity equation and Darcy's equation together with the fluid mass conservation in each of the $N$ pore components. Since only $x$ derivatives are involved, and all the quantities are macroscopic, we can simplify the writing by dropping the indices " $x^{\prime \prime}$ from $\nabla_{x}$ and " $s^{\prime \prime}$ from $\boldsymbol{U}_{s}^{(0)}$, all the superscripts (0), and the angle braces around $\left\langle\boldsymbol{W}^{i}\right\rangle$ and simply denote $\rho_{0}$ as $\rho_{f}$. Then

$$
\begin{aligned}
& \nabla \cdot \boldsymbol{D}_{\{4\}}: \boldsymbol{E}(\boldsymbol{U})+\sum_{i=1}^{N} \nabla \cdot \boldsymbol{\alpha}^{i} P^{i}=-\langle\rho\rangle \omega^{2} \boldsymbol{U}-\rho_{f} \omega^{2} \sum_{i=1}^{N} \boldsymbol{W}^{i}, \\
& i \omega \boldsymbol{W}^{i}=\frac{1}{\mu} \boldsymbol{K}^{i} \cdot\left(-\nabla P^{i}+\rho_{f} \omega^{2} \boldsymbol{U}\right), \quad i=1, \ldots, N, \quad \text { (39a) } \\
& \nabla \cdot \boldsymbol{W}^{i}=\boldsymbol{\alpha}^{i}: \boldsymbol{E}(\boldsymbol{U})+\sum_{j=1}^{N} B^{i j} P^{j}-\epsilon^{i} c_{f} P^{i}, \quad i=1, \ldots, N .
\end{aligned}
$$

\section{THE BIPHASIC MACROSCOPIC BEHAVIOR OF A SATURATED POROUS MEDIUM: DISCUSSION}

\section{A. Comparison with the single-pore, incompressible fluid case}

Let us start by examining how the macroscopic description, (39), differs from its counterpart in [7] as a consequence of the additional features which are taken into account here. First, two terms in the global dynamic equation, (39), become summations over the $N$ pore components, namely, the inertial term associated with the interstitial fluid, on the right-hand side, and the contribution of the pore pressures in the stress balance on the left-hand side.

Then a flow problem composed of the Darcy equation, (39b), and mass balance, (39), is associated with each of the $N$ pore components. Finally, the only effect of the fluid compressibility on this level of description is the introduction of the last term in (39). However, the structure of (39) is identical to that of its counterpart in [16], as shown by the following reformulation.

\section{B. Compact formulation of the equations}

By convention, the sets of quantities associated with the various pores, such as the pressures $P^{i}$, are gathered together in vectors denoted with an overbar. For instance,

$$
\overline{\boldsymbol{P}}=\left(P^{i}\right), \quad \overline{\boldsymbol{\alpha}}=\left(\boldsymbol{\alpha}^{i}\right) .
$$

Similarly, matrices denoted by two overbars are built by grouping the quantities associated with pairs of pores, e.g.,

$$
\overline{\bar{B}}=\left(B^{i j}\right)
$$


For convenience, the volume fraction $\epsilon^{i}$ and permeability $\boldsymbol{K}$ are stored in diagonal matrices rather than vectors,

$$
\overline{\bar{\epsilon}}=\left(\begin{array}{cccc}
\epsilon^{1} & & & \\
& \epsilon^{2} & & \\
& & \ddots & \\
& & & \epsilon^{N}
\end{array}\right), \quad \overline{\overline{\boldsymbol{K}}}=\left(\begin{array}{llll}
\boldsymbol{K}^{1} & & & \\
& \boldsymbol{K}^{2} & & \\
& & \ddots & \\
& & & \boldsymbol{K}^{N}
\end{array}\right) .
$$

In addition, $\bar{I}$ is the unit column vector.

Finally, we define an inner product " $\star$ " over the pore indices (superscripts), equivalent to the dot product "." over the spatial indices (subscripts). For instance, the summation on the left-hand side of (39) is

$$
\sum_{i=1}^{N} \boldsymbol{\alpha}^{i} P^{i}=\bar{\alpha} \star \bar{P} .
$$
form:

With these conventions, Eq. (39) can be written in matrix

$$
\begin{aligned}
& \nabla \cdot \boldsymbol{D}_{\{4\}}: \boldsymbol{E}(\boldsymbol{U})+\nabla \cdot \overline{\boldsymbol{\alpha}} \star \bar{P}=-\langle\rho\rangle \omega^{2} \boldsymbol{U}-\rho_{f} \omega^{2} \bar{I} \star \overline{\boldsymbol{W}} \\
& i \omega \overline{\boldsymbol{W}}=\frac{1}{\mu} \overline{\overline{\boldsymbol{K}}} \star\left(-\nabla \bar{P}+\rho_{f} \omega^{2} \overline{\boldsymbol{I}} \boldsymbol{U}\right), \\
& \nabla \cdot \overline{\boldsymbol{W}}=\overline{\boldsymbol{\alpha}}: \boldsymbol{E}(\boldsymbol{U})+\left(\overline{\bar{B}}-c_{f} \overline{\bar{\epsilon}}\right) \star \bar{P} .
\end{aligned}
$$

This formal rewriting makes apparent the one-by-one correspondence of all the terms in Eq. (44) and in its counterpart, (4.80) in [16], for an incompressible fluid in a totally connected pore space. It also makes some calculations much more tractable, such as the derivation of the Christoffel equation in Sec. V A.

\section{Effective coefficients: Meaning and associated closure problems}

The macroscopic description, (39) or (44), involves several effective coefficients. We summarize here their physical meaning and the way they can be determined from microscopic closure problems.

The elastic tensor $\boldsymbol{D}_{\{4\}}$ is the elastic stiffness tensor for the dry porous medium. $\boldsymbol{D}_{\{4\}}: \boldsymbol{E}$ is the mean stress tensor when a macroscopic strain $\boldsymbol{E}$ is imposed upon the medium. It can be determined by solving the local problem, Eq. (27), for a set of prescribed strains $\boldsymbol{E}$, in the absence of any pore pressure.

The tensors $\boldsymbol{\alpha}^{i}$ are defined by (30), and they represent the mean stress in the medium when it is submitted to a unit pressure $P^{i}=1$ in pore $i, P^{j}=0$ in the other pores $j \neq i$, and zero macroscopic strain $\boldsymbol{E}$. Accordingly, the associated closure problem is given by (29). Note that one such problem is to be solved for each of the $N$ pore components. Due to the Maxwell-Betti reciprocal theorem, $-\overline{\boldsymbol{\alpha}}: \boldsymbol{E}$ also represent the variations of the pore volumes in the dual problem where the dry medium is submitted to a macroscopic strain $\boldsymbol{E}$.

The opposite of the pore-interaction coefficient $B^{i j}$ defined by (31) is the variation of the pore volume $\Omega_{f}^{i}$ when a unit pressure is imposed in the pore $j$. They can be derived when problem (29) has been solved for the determination of $\boldsymbol{\alpha}^{i}$.
The dynamic permeability tensors $\boldsymbol{K}^{\boldsymbol{i}}$ are complex and frequency dependent. They are obtained for each of the percolating pore components, by solving the flow problem (33), for three directions of the macroscopic pressure gradient. Then $\boldsymbol{K}^{\boldsymbol{i}}$ results in the average (37), of the local tensor $\boldsymbol{k}^{i}$ (35).

All the closure problems associated with the elastic coefficients $\boldsymbol{D}_{\{4\}}, \boldsymbol{\alpha}^{i}$, and $B^{i j}$ can be solved numerically by using the $F M D$ solver of the Navier elastostatic equations described in [7] with prescribed pore pressures or macrostrain. FMD is based on a finite-volume formulation, in a structured or unstructured tetrahedral mesh.

The dynamic flow problems are solved by using the numerical code developed in Sec. IV.B in [7]. It also results from a finite-volume formulation, on the same kind of mesh.

\section{Effective coefficients: General properties}

We present here a list of general properties of the effective elastic coefficients. The detailed proofs are not given, but a hint about the method of demonstration is sometimes provided.

Of course, since $\boldsymbol{\alpha}^{i}$ is a stress tensor, it is symmetric. The Maxwell-Beti theorem can be used to show that the matrix $\overline{\bar{B}}$ is symmetric:

$$
B^{i j}=B^{j i} .
$$

$\overline{\bar{B}}$ has several other properties. $B^{i i}$ is negative since a positive pressure in $\Omega_{f}^{i}$ induces a positive change of this pore volume. The off-diagonal terms are generally negative (but this is not a theoretical requirement) and of a much smaller magnitude than the diagonal ones. The line sums of $\overline{\bar{B}}$ (sums of the components in a line if $\overline{\bar{B}}$ ) are negative (a positive pressure in a pore can only increase the total porosity).

The last property, together with the symmetry, implies that $\overline{\bar{B}}$ is diagonally dominant and, therefore, definite negative and invertible. Thus, $\overline{\bar{B}}-c_{f} \overline{\bar{\epsilon}}$ has the same properties, with the important consequence that its inverse $\overline{\bar{G}}$ can be defined for later use:

$$
\overline{\bar{G}}=\left(\overline{\bar{B}}-c_{f} \overline{\bar{\epsilon}}\right)^{-1} .
$$

Then the undrained stiffness tensor of the saturated medium is

$$
\boldsymbol{D}_{\{4\}}^{u}=\boldsymbol{D}_{\{4\}}-\overline{\boldsymbol{\alpha}} \star \overline{\bar{G}} \star \overline{\boldsymbol{\alpha}} .
$$

This can be shown by considering a static load $(\omega=$ $0)$ without fluid flow $(\overline{\boldsymbol{W}}=0)$. Then $\bar{P}$ can be obtained from $(44 c)$ :

$$
\bar{P}=-\overline{\bar{G}} \star \overline{\boldsymbol{\alpha}}: \boldsymbol{E} .
$$

Injecting this into (44a) yields the static undrained equilibrium equation

$$
\nabla \cdot \boldsymbol{D}_{\{4\}}^{u}: \boldsymbol{E}=0 .
$$

Additional properties can be stated, based on a superposition argument, if the solid elastic properties are uniform with bulk and shear moduli $K_{s}$ and $\mu_{s}$. Then the trace of $\overline{\boldsymbol{\alpha}}$ is

$$
{ }^{t r} \bar{\alpha}=3 \bar{I} \star\left(K_{s} \overline{\bar{B}}-\overline{\bar{\epsilon}}\right)
$$


and

$$
\boldsymbol{I}+\bar{I} \star \overline{\boldsymbol{\alpha}}=\frac{1}{3 K_{s}}\left(\boldsymbol{D}_{\{4\}}: \boldsymbol{I}\right) .
$$

If the porous medium is macroscopically isotropic, with bulk modulus $K_{\text {eff }}$, the last property reads

$$
\bar{I} \star \overline{\boldsymbol{\alpha}}=\left(\frac{K_{\mathrm{eff}}}{K_{s}}-1\right) \boldsymbol{I},
$$

and if the solid material is isotropic, with $\boldsymbol{C}_{\{4\}}: \boldsymbol{I}=3 K_{s}$,

$$
\bar{I} \star \overline{\boldsymbol{\alpha}}=\frac{1}{3 K_{s}}\left(\boldsymbol{D}_{\{4\}}-\boldsymbol{C}_{\{4\}}\right): \boldsymbol{I} .
$$

Finally, useful orders of magnitude for the $B^{i j}$ coefficients can be obtained in simple model situations. For noninteracting spherical pores

$$
B^{i i}=-\frac{3}{4 \mu_{s}} \epsilon^{i},
$$

and for slender cylindrical pores

$$
B^{i i}=-\frac{1}{2 \mu_{s}} \epsilon^{i} .
$$

If it is assumed that tubes and spheres are limiting aspects of the pore morphology in consolidated, moderately porous media, this suggests that these two values are practical lower and upper bounds for $B^{i j}$. This is well supported by all the numerical results of [7] for reconstructed unimodal and bimodal media.

Finally, a leading-order estimate of the off-diagonal $B^{i j}$ for semidilute dispersion of spherical cavities can be obtained by regarding cavity $j$ in the strain field induced at its center by a unit pressure in cavity $i$ :

$$
B^{i j}=\frac{3}{4 \mu_{s}}\left(1+\frac{3 K_{s}}{4 \mu_{s}}\right) \epsilon^{i} \epsilon^{j} \quad(i \neq j) .
$$

It is of opposite sign and of a much smaller magnitude than $B^{i j}$ in (54).

\section{PLANE WAVES}

Consider now the propagation of harmonic plane waves along direction $\boldsymbol{p}$. Hence, we look for solutions of the poroelastic equation, (44), with the forms

$$
\begin{aligned}
& \boldsymbol{u}=\boldsymbol{U} e^{i \omega t}=\hat{\boldsymbol{U}} e^{i(\omega t-k x \cdot p)} \\
& \boldsymbol{w}=\boldsymbol{W} e^{i \omega t}=\hat{\boldsymbol{W}} e^{i(\omega t-k x \cdot p)}, \\
& p=P e^{i \omega t}=\hat{P} e^{i(\omega t-k x \cdot p)},
\end{aligned}
$$

where $k$ is the wave number with $c=\frac{\omega}{k}$.

Generally, the velocity $c$ is complex, i.e.,

$$
c=c_{r}+i c_{i}, \quad c_{r}=\operatorname{Re}(c), \quad c_{i}=\operatorname{Im}(c) .
$$

Inserting Eq. (58) into Eq. (57) yields

$$
\boldsymbol{u}=\boldsymbol{U} e^{i \omega t}=\hat{\boldsymbol{U}} e^{i \omega\left(t-\frac{c_{r}}{|c|^{2}} \boldsymbol{x} \cdot \boldsymbol{p}\right)} e^{-\omega \frac{c_{r}}{|c|^{2}} \boldsymbol{x} \cdot \boldsymbol{p}} .
$$

It is obvious that the velocity and the attenuation coefficient of the wave are

$$
c_{p}=\frac{|c|^{2}}{c_{r}}, \quad a_{h}=\frac{\omega c_{i}}{|c|^{2}} .
$$

Equivalently, the wave attenuation can be quantified by the penetration depth $h$, which is related to the attenuation coefficient by

$$
h=a_{h}^{-1}=\frac{|c|^{2}}{\omega c_{i}} .
$$

\section{A. Christoffel equation}

The $\boldsymbol{x}$ derivative simply corresponds to a multiplication by $-i k \boldsymbol{p}$. Hence, the pressure can be obtained from (44c) as

$$
\bar{P}=\overline{\bar{G}} \star[\nabla \cdot \overline{\boldsymbol{W}}-\overline{\boldsymbol{\alpha}}: \boldsymbol{E}(\boldsymbol{U})]
$$

or

$$
\hat{\bar{P}}=-i k \overline{\bar{G}} \star[\boldsymbol{p} \cdot \hat{\boldsymbol{W}}-\overline{\boldsymbol{\alpha}}: \boldsymbol{p} \hat{\boldsymbol{U}}] .
$$

When injecting this into (44a) and (44b), the following eigenvalue problem is obtained, which is formally identical to the one in [7] and [16] for a single-pore component and an incompressible fluid:

$$
\begin{aligned}
& \left(\begin{array}{lr}
\boldsymbol{p} \cdot \boldsymbol{D}_{\{4\}}^{u} \cdot \boldsymbol{p} & \boldsymbol{p} \cdot \overline{\boldsymbol{\alpha}} \star \overline{\bar{G}} \boldsymbol{p} \star \\
-\frac{1}{\mu_{f}} \boldsymbol{p} \cdot \overline{\overline{\boldsymbol{K}}} \star \overline{\bar{G}} \star \overline{\boldsymbol{\alpha}} \cdot \boldsymbol{p} \cdot & \frac{1}{\mu_{f}} \boldsymbol{p} \cdot \overline{\overline{\boldsymbol{K}}} \star \overline{\bar{G}} \boldsymbol{p} \star
\end{array}\right) \cdot\left(\begin{array}{l}
\hat{\boldsymbol{U}} \\
\hat{\boldsymbol{W}}
\end{array}\right) \\
& =c^{2}\left(\begin{array}{lr}
\langle\rho\rangle & \rho_{f} \bar{I} \star \\
-\frac{\rho_{f}}{\mu_{f}} \overline{\overline{\boldsymbol{K}}} \star \bar{I} . & \frac{i}{\omega}
\end{array}\right) \cdot\left(\begin{array}{c}
\hat{\boldsymbol{U}} \\
\hat{\hat{\boldsymbol{W}}}
\end{array}\right) .
\end{aligned}
$$

Note that this is a fully general formulation, for an arbitrary number $N$ of pore components. However, it should be noted that two independent percolating pore systems in the same material are extremely uncommon. In practice, only two situations occur. In the first one, there is no percolating pore component. In the second one, a single percolating component exists, which is referred to by $i=1$, together with several closed ones $(i=2, \ldots, N)$.

In the first case, all the permeabilities $\boldsymbol{k}^{i}$ and fluxes $\hat{\boldsymbol{W}}^{i}$ are 0 , and (63) reduces to

$$
\boldsymbol{p} \cdot \boldsymbol{D}_{\{4\}}^{u}: \boldsymbol{p} \hat{\boldsymbol{U}}=c^{2}\langle\rho\rangle \hat{\boldsymbol{U}} .
$$

In the second case, only $\boldsymbol{K}^{1}$ and $\hat{\boldsymbol{W}}^{1}$ are nonzero. Denote by $\bar{G}$ and $G$ the first column and the first diagonal element of $\overline{\bar{G}}$ :

$$
\bar{G}=\left(G^{1 i}\right) \quad G=G^{11} .
$$

Then (63) reduces to

$$
\begin{gathered}
\left(\begin{array}{lr}
\boldsymbol{p} \cdot \boldsymbol{D}_{\{4\}}^{u} \cdot \boldsymbol{p} & \boldsymbol{p} \cdot \overline{\boldsymbol{\alpha}} \star \bar{G} \boldsymbol{p} \cdot \\
-\frac{1}{\mu_{f}} \boldsymbol{p} \cdot \boldsymbol{K}^{1} \bar{G} \star \overline{\boldsymbol{\alpha}} \cdot \boldsymbol{p} \cdot & \frac{1}{\mu_{f}} \boldsymbol{p} \cdot \boldsymbol{K}^{1} G \boldsymbol{p} \cdot
\end{array}\right) \cdot\left(\begin{array}{c}
\hat{\boldsymbol{U}} \\
\hat{\boldsymbol{W}}^{1}
\end{array}\right) \\
=c^{2}\left(\begin{array}{cc}
\langle\rho\rangle & \rho_{f} \\
-\frac{\rho_{f}}{\mu_{f}} \boldsymbol{K}^{1} \cdot & \frac{i}{\omega}
\end{array}\right) \cdot\left(\begin{array}{c}
\hat{\boldsymbol{U}} \\
\hat{\boldsymbol{W}}^{1}
\end{array}\right) .
\end{gathered}
$$

Equation (66) is simpler than Eq. (63) in the sense that it involves a single vector equation for $\hat{\boldsymbol{W}}^{1}$ instead of $N$ for $\hat{\boldsymbol{W}}$. 


\section{B. Full analytical solution in the macroscopically isotropic case}

For illustration, and to identify some general trends, we present here full analytical solutions when all macroscopic effective tensors are isotropic. For instance, $\boldsymbol{K}^{1}$ and $\boldsymbol{\alpha}^{i}$ are spherical,

$$
\boldsymbol{K}^{1}=K \boldsymbol{I}, \quad \boldsymbol{\alpha}^{i}=\alpha^{i} \boldsymbol{I}, \quad i=1, \ldots, N,
$$

where $\boldsymbol{I}$ is the unit tensor. Consequently, the groups $\bar{G} \star \overline{\boldsymbol{\alpha}}$ which appear in (66) are spherical:

$$
\overline{\boldsymbol{\alpha}} \star \bar{G}=\bar{G} \star \bar{\alpha} \boldsymbol{I}, \quad \overline{\boldsymbol{\alpha}} \star \overline{\bar{G}} \star \overline{\boldsymbol{\alpha}}=\bar{\alpha} \star \overline{\bar{G}} \star \bar{\alpha} \boldsymbol{I I} .
$$

The last property implies that the effective Lamé coefficients associated with the isotropic stiffness tensors $\boldsymbol{D}_{\{4\}}$ and $\boldsymbol{D}_{\{4\}}^{u}$ for the dry and saturated porous media are related by

$$
\lambda_{\mathrm{eff}}^{u}=\lambda_{\mathrm{eff}}^{0}-\bar{\alpha} \star \overline{\bar{G}} \star \bar{\alpha}, \quad \mu_{\mathrm{eff}}^{u}=\mu_{\mathrm{eff}}^{0} .
$$

Note that (67) is a very strong and often unrealistic hypothesis. However, global isotropy of $\boldsymbol{D}_{\{4\}}^{u}$ only requires that the combination $\bar{\alpha} \star \overline{\bar{G}} \star \bar{\alpha}$ is isotropic, which can result from the statistical averaging of many anisotropic, but randomly oriented individual pore contributions. This happens, for instance, when a solid matrix, with Lamé coefficients $\lambda_{s}$ and $\mu_{s}$ and Poisson ratio $\nu_{s}$, contains a dilute dispersion of penny-shaped microcracks with radius $R$ and aperture $b \ll R$, randomly oriented with normal vectors $\boldsymbol{n}^{i}$. The cracks do not interact in the dilute limit, and therefore $\overline{\bar{B}}$ and $\overline{\bar{G}}$ are diagonal. The coefficients $B^{i i}$ and the tensors $\boldsymbol{\alpha}^{i}$ can be deduced from earlier results in [18] and [19]. They are given by

$$
B^{i i}=-\frac{8}{3} \frac{R}{\pi b} \frac{1-v_{s}}{\mu_{s}} \epsilon^{i}, \quad \boldsymbol{\alpha}^{i}=B^{i i}\left[\lambda_{s} \boldsymbol{I}+2 \mu_{s} \boldsymbol{n}^{i} \boldsymbol{n}^{i}\right] .
$$

Although the individual $\boldsymbol{\alpha}^{i}$ tensors are anisotropic, the summation of many uncorrelated contributions without preferential orientation makes $\overline{\boldsymbol{\alpha}} \star \overline{\bar{G}} \star \overline{\boldsymbol{\alpha}}$ isotropic, as well as $\boldsymbol{D}_{\{4\}}^{u}$, in view of (47). Its associated Lamé coefficients can be obtained as

$$
\begin{aligned}
\lambda_{\mathrm{eff}}^{u} & =\lambda_{\mathrm{eff}}^{0}+\frac{1+6 v_{s}-v_{s}^{2}}{2 v_{s}\left(1-2 v_{s}\right)} M \lambda_{s} \epsilon, \\
\mu_{\mathrm{eff}}^{u} & =\mu_{\mathrm{eff}}^{0}+M \mu_{s} \epsilon, \quad \text { with } \\
M & =\frac{\frac{32}{45}\left(1-v_{s}\right) \frac{R}{\pi b}}{1+\frac{3}{8} \frac{\mu_{s} c_{f}}{1-v_{s}} \frac{\pi b}{R}} .
\end{aligned}
$$

A similar result is obtained if the cracks are thin oblate ellipsoids with semiaxes $(R, R, b / 2)$, with $b$ replaced by $2 b / 3$ in (70) and (71) [20]. Thus, whereas the undrained effective shear modulus is unaffected by the presence of the intersticial fluid in (69) when the $\boldsymbol{\alpha}^{i}$ values are isotropic, for instance, for spherical inclusions, $\mu_{\text {eff }}^{u}$ differs from $\mu_{\text {eff }}^{0}$ when the inclusions are anisotropic.

For simplicity, the stonger form, (67), of the isotropy hypothesis is used in the remainder of this section.

\section{No percolating pore components}

The Christoffel equation reduces to (64). Solutions exist with $\hat{U}$ orthogonal (shear waves) or parallel (compression wave) to $\boldsymbol{p}$. For the shear wave, with $\hat{\boldsymbol{U}} \perp \boldsymbol{p}$,

$$
\hat{P}_{\perp}=0, \quad \hat{W}=0, \quad c_{\perp}^{2}=\frac{\mu_{\text {eff }}^{u}}{\langle\rho\rangle} .
$$

There is no fluid flow and no induced pore pressure and the wave velocity depends on the effective parameters $\mu_{\text {eff }}^{u}$ and $\langle\rho\rangle$, according to the same formula as in uniform continuous materials. Note that when (69) applies, $c_{\perp}$ is not affected by the presence of an interstitial fluid, aside from its contribution to $\langle\rho\rangle$.

For the compression wave, with $\hat{\boldsymbol{U}}=\hat{U} \boldsymbol{p}$,

$$
\hat{\bar{P}}_{\|}=i k \overline{\bar{G}} \star \bar{\alpha} \hat{U}, \quad \hat{\bar{W}}=0, \quad c_{\|}^{2}=\frac{\lambda_{\text {eff }}^{u}+2 \mu_{\mathrm{eff}}^{u}}{\langle\rho\rangle} .
$$

There is no fluid flow since all the pores are closed, but there is a pore pressure proportional to the wave amplitude $\hat{U}$. Again, the wave velocity is given by the usual formula in terms of the effective elastic constants and density. There is no imaginary component in $c_{\|}$, i.e., no attenuation, but it is influenced by the presence of the interstitial fluid.

\section{One percolating pore component}

For shear waves, with $\hat{\boldsymbol{U}}$ and $\hat{\bar{W}}$ orthogonal to $\boldsymbol{p}$, the Christoffel equation, (66), reduces to

$$
\begin{aligned}
\mu_{\mathrm{eff}}^{u} \hat{\boldsymbol{U}} & =c_{\perp}^{2}\left[\langle\rho\rangle \hat{\boldsymbol{U}}+\rho_{f} \hat{\boldsymbol{W}}^{1}\right], \\
0 & =-\frac{\rho_{f}}{\mu_{f}} K \hat{\boldsymbol{U}}+\frac{i}{\omega} \hat{\boldsymbol{W}}^{1} .
\end{aligned}
$$

The pore pressures $\hat{\bar{P}}$ are 0 in view of (62b). The fluid flow rate is obtained from (74b) and is proportional to the wave amplitude $\hat{\boldsymbol{U}}$ :

$$
\hat{\boldsymbol{W}}^{1}=-\frac{i \omega \rho_{f} K}{\mu_{f}} \hat{\boldsymbol{U}}
$$

Injecting this into (74 a) yields the shear wave velocity,

$$
c_{\perp}^{2}=\frac{\mu_{\mathrm{eff}}^{u}}{\langle\rho\rangle\left[1-i \frac{K \rho_{f}^{2} \omega}{\mu_{f}\langle\rho\rangle}\right]} .
$$

Let $F$ denote the following dimensionless number:

$$
F=\frac{K \rho_{f}^{2} \omega}{\mu_{f}\langle\rho\rangle}
$$

$F$ is always very small, in the practical range of the physical parameters. This property can be used to rewrite (76) as

$$
c_{\perp}^{2}=\frac{\mu_{\mathrm{eff}}^{u}}{\langle\rho\rangle}+i \frac{K}{\mu_{f}} \frac{\rho_{f}^{2} \mu_{\mathrm{eff}}^{u}}{\langle\rho\rangle^{2}} \omega+O\left(F^{2}\right) .
$$

Hence, the interstitial fluid slows down the shear wave because of its contribution to $\langle\rho\rangle$, but it also introduces an imaginary part in $c_{\perp}$, i.e., an attenuation which does not exist in (72) when the pores are closed.

The other solutions of (66) are for $\hat{\boldsymbol{U}}=\hat{U} \boldsymbol{p}, \hat{\boldsymbol{W}}^{1}=\hat{W} \boldsymbol{p}$, i.e., for compression waves. The Christoffel equation, (66), reads

$$
\left(\begin{array}{ll}
\lambda_{\mathrm{eff}}^{u}+2 \mu_{\mathrm{eff}}^{u}-\langle\rho\rangle c_{\|}^{2} & \bar{\alpha} \star \bar{G}-\rho_{f} c_{\|}^{2} \\
-\frac{K}{\mu_{f}}\left[\bar{\alpha} \star \bar{G}-\rho_{f} c_{\|}^{2}\right] & G \frac{K}{\mu_{f}}-\frac{i}{\omega} c_{\|}^{2}
\end{array}\right)\left(\begin{array}{c}
\hat{U} \\
\hat{W}
\end{array}\right)=0 .
$$



tion

Equating the determinant to 0 yields the biquadratic equa-

$$
\begin{gathered}
(1-i F) c_{\|}^{4}-\frac{\lambda_{\mathrm{eff}}^{u}+2 \mu_{\mathrm{eff}}^{u}}{\langle\rho\rangle}(1-i F Z) c_{\|}^{2} \\
-i\left(\frac{\lambda_{\mathrm{eff}}^{u}+2 \mu_{\mathrm{eff}}^{u}}{\langle\rho\rangle}\right)^{2} F Y=0,
\end{gathered}
$$

with

$$
\begin{aligned}
& Z=\frac{\left(G\langle\rho\rangle+2 \bar{\alpha} \star \bar{G} \rho_{f}\right)\langle\rho\rangle}{\left(\lambda_{\mathrm{eff}}^{u}+2 \mu_{\mathrm{eff}}^{u}\right) \rho_{f}^{2}}, \\
& Y=\frac{\langle\rho\rangle^{2}}{\rho_{f}^{2}} \frac{\left(\lambda_{\mathrm{eff}}^{u}+2 \mu_{\mathrm{eff}}^{u}\right) G+(\bar{\alpha} \star \bar{G})^{2}}{\left(\lambda_{\mathrm{eff}}^{u}+2 \mu_{\mathrm{eff}}^{u}\right)^{2}} .
\end{aligned}
$$

The solution to (80) is

$$
c_{\|}^{2}=\frac{\lambda_{\mathrm{eff}}^{u}+2 \mu_{\mathrm{eff}}^{u}}{\langle\rho\rangle} \frac{1-i F Z}{2(1-i F)}\left[1 \pm \sqrt{1+4 i F Y \frac{1-i F}{(1-i F Z)^{2}}}\right] .
$$

The plus and minus signs yield the velocities of the fast and slow compression waves, respectively. In both cases, the fluid displacement and the pressures are given by

$$
\begin{aligned}
& \hat{W}=i \omega \frac{K \rho_{f}}{\mu_{f}} \frac{c_{\|}^{2}-\bar{\alpha} \star \bar{G} / \rho_{f}}{c_{\|}^{2}+i \omega G K / \mu_{f}} \hat{U}, \\
& \hat{\bar{P}}=\frac{i \omega}{c_{\|}}(\overline{\bar{G}} \star \bar{\alpha} \hat{U}-\bar{G} \hat{W}) .
\end{aligned}
$$

Expression (82) for $c_{\|}$is exact, but an approximate one, in the form of a first-order expansion in terms of a small number $F$, is more enlightening, since it makes it easier to identify the contributions of the fluid flow and compressibility effects.

For the fast compression wave,

$$
\begin{aligned}
c_{\|}^{2}= & \frac{\lambda_{\text {eff }}^{u}+2 \mu_{\mathrm{eff}}^{u}}{\langle\rho\rangle}\left[1+i \frac{K \omega}{\mu_{f}} \frac{\rho_{f}^{2}}{\langle\rho\rangle}\left(1-\frac{\langle\rho\rangle(\bar{\alpha} \star \bar{G})}{\rho_{f}\left(\lambda_{\mathrm{eff}}^{u}+2 \mu_{\mathrm{eff}}^{u}\right)}\right)^{2}\right] \\
& +O\left(F^{2}\right) .
\end{aligned}
$$

If $F$ vanishes (low frequency $\omega$ or permeability $K / \mu_{f}$ ), Eq. (84) reduces to (73). The wave velocity is influenced by the fluid compressibility through (69), but not by the fluid flow, and it is real (no attenuation).

If the fluid is very compressible $\left(c_{f} \rightarrow \infty, \bar{\alpha} \star \bar{G} \rightarrow 0\right)$, the wave velocity depends on the dry elastic moduli, but the flow introduces an attenuation,

$$
c_{\|}^{2}=\frac{\lambda_{\mathrm{eff}}^{0}+2 \mu_{\mathrm{eff}}^{0}}{\langle\rho\rangle}\left[1+i \frac{K \omega}{\mu_{f}} \frac{\rho_{f}^{2}}{\langle\rho\rangle}\right]+O\left(F^{2}\right) .
$$

Recall that $K$ is also complex and frequency dependent.

For the slow compression wave,

$$
c_{\|}^{2}=-i \frac{K \omega G}{\mu_{f}}\left[1+\frac{(\bar{\alpha} \star \bar{G})^{2}}{G\left(\lambda_{\mathrm{eff}}^{u}+2 \mu_{\mathrm{eff}}^{u}\right)}\right]+O\left(F^{2}\right) .
$$

Note that this velocity does not depend on the fluid density. The fluid displacement amplitude is obtained from (83) and (86) as

$$
\hat{W}=\frac{\lambda_{\text {eff }}^{u}+2 \mu_{\mathrm{eff}}^{u}}{\bar{\alpha} \star \bar{G}}\left(1-\frac{\rho_{f} c_{\|}^{2}}{\bar{\alpha} \star \bar{G}}\right) \hat{U}+O\left(F^{2}\right) .
$$

The prefactor is real, positive, and of order $O(1)$. The corrective term in parentheses is complex [see Eq. (86)] but of order $O(F)$. Hence, $\hat{W}$ is in phase with $\hat{U}$ and of the same order of magnitude.

\section{APPLICATIONS}

This section has two purposes. First, a synthetic view of the results is presented together with a reminder of the numerical techniques. Second, systematic applications are made to a synthetic, but realistic, porous medium in order to illustrate the methodology.

\section{A. Requirements for a complete solution and numerical techniques}

The solution of (63) or (66), or more generally of the poroelastic equation (44c), requires the knowledge of the following quantities. The dry medium elastic tensor $\boldsymbol{D}_{\{4\}}$ can be obtained by solving six elastostatic problems (see Sec. IV D). The permeability $\overline{\overline{\boldsymbol{K}}}$, which generally reduces to only $\boldsymbol{K}^{i}$, is derived from three flow problems with a macroscopic pressure gradient aligned with the three axes, successively; since $\boldsymbol{K}^{1}$ is frequency dependent, these flow problems have to be solved for all the investigated values of $\omega$. The full knowledge of the interaction matrix $\overline{\bar{B}}$ is required, even in the case of only one or even zero percolating pore components, because $\overline{\bar{G}}$ and $\boldsymbol{D}_{\{4\}}^{u}$ [see Eqs. (46) and (47)] need to be evaluated; this necessitates the solution of an elastic problem with a unit pressure imposed successively in each of the $N$ pore components and may become a problem since $N$ can possibly be very large. Various strategies are possible to devise sensible approximations in such situations. They are presented and tested in Sec. VIB.

Note that the fluid compressibility effects play a role only in $\boldsymbol{D}_{\{4\}}^{u}$ through $\overline{\bar{G}}$ [see Eqs. (46) and (47)] and that they do not require the solution of any additional closure problem. It seems also possible to reduce the computational cost by calculating only the components of the effective coefficients needed for a specified direction of propagation $\boldsymbol{p}$. For instance, calculating $\boldsymbol{p} \cdot \boldsymbol{K}^{1}$ requires only one flow problem solution (per frequency) instead of three for the full $\boldsymbol{K}^{1}$. Similarly, $\boldsymbol{p} \cdot \boldsymbol{D}_{\{4\}} \cdot \boldsymbol{p}$ can be determined from only three solutions (one compression and two shears), instead of six for the full $\boldsymbol{D}_{\{4\}}$.

However, one should be very cautious about such shortcuts. In general, $\boldsymbol{D}_{\{4\}}, \boldsymbol{D}_{\{4\}}^{u}, \boldsymbol{\alpha}^{i}$, and $\boldsymbol{K}^{i}$ are anisotropic tensors. In addition, there is no reason a priori for their principal directions to be aligned and, therefore, no way to set $\boldsymbol{p}$ in a direction corresponding to an eigenvector for all of them. This means, for instance, that the vector $\hat{U}$ solution to (63) is a priori not aligned with $\boldsymbol{p}$ and thus that the knowledge of $\boldsymbol{p} \cdot \boldsymbol{K}^{1}$ is not sufficient to evaluate $\boldsymbol{K}^{1} \cdot \hat{U}$ on the right-hand side of (66). Such problems occur of course for several other terms in (63) or (66).

The numerical techniques to discretize and solve these equations are the same as the ones described and used in [7]; therefore, it is not necessary to detail them again. The porous medium is usually meshed by a structured or unstructured tetrahedral mesh. In the present work, all the calculations have been made with the SCT6-structured mesh. Then the studied 


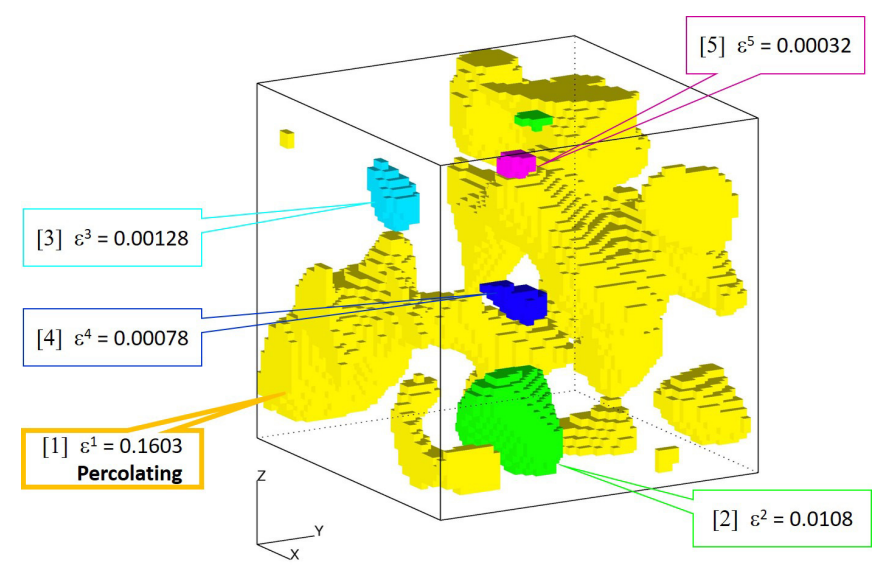

FIG. 1. The pore space of the test sample. The volume fractions $\epsilon^{i}$ are shown for each of the five components.

equation is discretized by a finite-volume technique which turns out to be efficient for the elasticity equation as well. The resulting linear system is solved by a conjugate gradient algorithm.

\section{B. Full treatment of a sample case 1. Introduction}

The detailed treatment of a test case yields three objectives, namely, to illustrate all the aspects of the previous theoretical developments, to check both the theoretical predictions and the numerical solvers and procedures, and, finally, to present and test various strategies for a simplified approximate procedure when the number of pore components is large. The medium is made of elementary cubes of size $a$ which are either void or solid. The overall dimensions of the cubic unit cell are $48 a \times 48 a \times 48 a$. The medium is computer generated according to the scheme described in [21]. An uncorrelated normal field $X(\boldsymbol{x})$ is generated. Then it is correlated by the covariance function $\exp \left(-r^{2} / L_{c}^{2}\right)$, where $L_{c}$ is the correlation length which is chosen to be equal to $8 a$. The corresponding normal field $Y(\boldsymbol{x})$ is thresholded to get void voxels which correspond to pores with a probability $\epsilon=0.1735$. The pore space consists in $N=5$ pore components, shown in Fig. 1 together with their volume fractions. The largest one, with $\epsilon^{1}=0.16$, percolates along all directions of space and the four others are closed. One of them has a quite significant volume fraction, $\epsilon^{2}=0.011$. The three others are smaller, but their cumulated volume is still to be taken into consideration, since $\epsilon^{3}+\epsilon^{4}+\epsilon^{5}=0.0024$. The pores are filled with a compressible fluid, while the solid phase has a Young modulus $E_{s}$ and a Poisson ratio $v_{s}$.

\section{Effective elastic properties}

The effective elastic coefficients of this porous medium are calculated for a solid with a Poisson ratio $v_{s}=0.30$. The numerical values are given in Appendix A and only the main conclusions are presented here.

$\boldsymbol{D}_{\{4\}}$ and the tensors $\boldsymbol{\alpha}^{i}$ are provided in Sec. A 1. $\boldsymbol{D}_{\{4\}}$ was obtained from the stress field calculated when successively imposing three compression and three shear macroscopic strain fields. It should be perfectly symmetric; therefore, the very small differences between corresponding $D_{k l}$ and $D_{l k}$ components are a measure of the accuracy of the numerical calculations. The medium is approximately but not perfectly isotropic. It is slightly stiffer along the $y$ direction.

The tensors $\boldsymbol{\alpha}^{i}$ have been computed independently by imposing a unit pressure in each pore and measuring the resulting mean stress. The property, (51), which is valid for uniform solid properties, is accurately verified.

All the $\boldsymbol{\alpha}^{i}$ are nearly spherical. Their mean trace components is roughly proportional to the volume fraction

$$
\alpha^{1} / \epsilon^{1} \approx 2.5, \quad \alpha^{i} / \epsilon^{i} \approx 2.2 \pm 0.1, \quad i=2, \ldots, 5 .
$$

The full matrix $\overline{\bar{B}}$ was calculated by imposing a unit pressure in each of the pores and measuring the changes in all the pore volumes. It is given in Sec. A 2. It is symmetric, as theoretically predicted, and the property (50) is verified. $\overline{\bar{B}}$ is diagonally dominant. The small pores $(i>1)$ do not significantly interact. The only terms of nonvanishing magnitude are diagonal, and in the first line or row, i.e., interaction coefficients between the large pore component 1 and the other small ones.

Matrix $\overline{\bar{B}}$ was approximated in various ways that are summarized here. It was first compared to the prediction $\overline{\bar{B}}_{\text {sph }}$ based on (54) and (56), which assumes spherical pores and involves only the volume fractions. Despite the very crude character of this approximation, the relative errors are within $26 \%$ for all the terms of significant magnitude.

Other approximations were tried, and the most successful necessitates another elastic solution. Get first the line sums of $\bar{B}$ from (50) if the solid is uniform or with $P=1$ in all the pores otherwise; put them on the diagonal. Then solve a problem with a unit pressure in pore component 1 . The coefficients $B^{1 i}$ are obtained from the pore volume changes. Hence, the first line and row are known directly. Put on the diagonal for $i>1$ the remaining line sums. This is equivalent to accounting for the interactions between pore 1 and the others but neglecting the interactions between small pores. In other words, we focus on the components of $\overline{\bar{B}}$ which were found to be of significant magnitude. This yields an excellent approximation for the significant terms of $\overline{\bar{B}}$ since the absolute error never exceeds $1.5 \times 10^{-4}$. Furthermore, the most important terms in $\overline{\bar{B}}$ associated with the pore which may carry fluid are calculated exactly.

\section{Dynamic permeability}

The full dynamic permeability tensor was calculated for four values of the dimensionless frequency

$$
\omega_{c}=\frac{\rho_{f} \Lambda^{2} \omega}{\mu_{f}} .
$$

The Johnson length $\Lambda$ [22] is an intrinsic measure of the interconnected pore size defined as

$$
\Lambda=2 \frac{\int_{\Omega}|\nabla \psi(\boldsymbol{r})|^{2} d^{3} r}{\int_{S}|\nabla \psi(\boldsymbol{r})|^{2} d^{2} s},
$$

where $\psi(\boldsymbol{r})$ is the solution of the Laplace equation in the pore space. It is obtained by a separate simulation of the electrical conductivity problem with a mean gradient set along 
the direction $\boldsymbol{p}$. The values for $\boldsymbol{p}$ along $x, y$, and $z$ are very similar, in agreement with the isotropy of the imaginary part of $\boldsymbol{K}$ at high frequency [see Eq. (B4)], and their average is used in (89) for simplicity. $\Lambda$ was shown in [22] to be a governing parameter for the dynamic permeability, and the normalization, (89), was indeed found to successively unify numerical results for a variety of media in [7], [23], and [24].

The full numerical results are provided in Appendix B. Since only pore 1 is percolating, $\boldsymbol{K}$ corresponds to $\boldsymbol{K}^{1}$. The values for $\boldsymbol{K}$ are given by (B1) and they are symmetric as they should be. The evolution of $\boldsymbol{K}$ with the frequency complies with the general features predicted by [22], complicated by its anisotropic character. Although the sample is built in a statistically isotropic way, it is somewhat anisotropic because of its small size, and accordingly, $\boldsymbol{K}$ is found anisotropic for $\omega=0$. Of course, it is real. For high frequencies, $\boldsymbol{K}$ tends to become purely imaginary and approaches isotropy. However, in a range of intermediate frequencies, both real and imaginary parts of $\boldsymbol{K}$ have significant magnitudes. Furthermore, its eigenvalues and eigenvectors are complex, which means that in the eigenmodes, the fluid velocity and pressure gradient rotate during a period.

\section{Solutions of the Christoffel equation}

Since the effective tensors $\boldsymbol{D}_{\{4\}}, \boldsymbol{\alpha}^{i}, \overline{\bar{B}}$, and $\boldsymbol{K}$ have been fully determined, the acoustic properties of the synthetic sample can be calculated by solving the Christoffel equation, (66). The physical constants for the solid and fluid are taken as follows:

$$
\begin{aligned}
& E_{s}=70 \mathrm{GPa}, \quad \nu_{s}=0.30, \quad \rho_{s}=2700 \mathrm{~kg} \cdot \mathrm{m}^{-3}, \\
& c_{f}=5 \times 10^{-10} \mathrm{~Pa}^{-1}, \quad \mu_{f}=10^{-3} \mathrm{~Pa} \cdot \mathrm{s}, \\
& \rho_{f}=1000 \mathrm{~kg} \cdot \mathrm{m}^{-3} .
\end{aligned}
$$

When faced with an anisotropic material, the usual approach is to determine first the acoustic properties for a wave propagation along the material principal directions. If solutions for compression and shear waves can be obtained, meaning with a displacement parallel or orthogonal to the propagation direction, the behavior along the oblique directions can be deduced from these canonical data.

This approach is possible for a dry medium, since only the stiffness tensor $\boldsymbol{D}_{\{4\}}$ can be anisotropic. But it is generally impossible for a saturated medium since then $\boldsymbol{D}_{\{4\}}^{u}, \overline{\boldsymbol{\alpha}}, \overline{\boldsymbol{\alpha}} \star \overline{\bar{G}}$, and $\boldsymbol{K}$ can be anisotropic, with no reason a priori for their principal directions to be aligned. Furthermore, as demonstrated in the previous subsection, the eigenvectors of $\overline{\boldsymbol{K}}$ can be complex, which means that their direction rotates during a period.

Therefore, there is no way to set the propagation along a direction $\boldsymbol{p}$ where the existence of pure compression and a shear wave is expected, and in general such a direction does not exist. This sheds a disturbing light on the interpretation and use of acoustic data.

For these reasons, we consider wave propagation along directions arbitrarily aligned with the $x, y$, and $z$ axes. The solutions of the Christoffel equation obtained in the most complex situation of the intermediate frequency $\omega_{c}=10$ are thoroughly discussed in Appendix C.

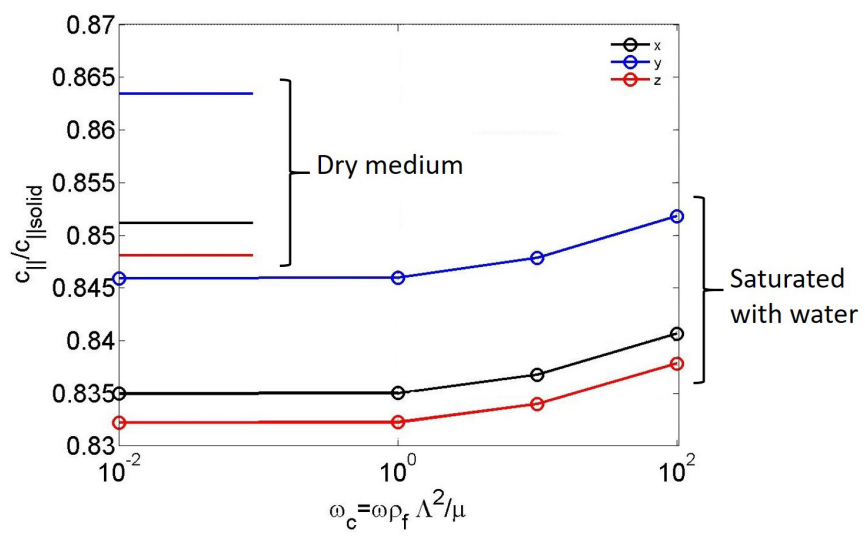

FIG. 2. The fast compression wave velocity, normalized by the velocity in the solid as a function of the dimensionless frequency $\omega_{c}$ calculated along the directions $x, y$, and $z$.

In summary, it is shown that even in the most complex situation where the principal directions of the effect tensors are not aligned, and in the range of frequencies where the eigenvectors of $\boldsymbol{K}$ are complex, the solutions obtained for propagation along an arbitrary direction can be unambiguously identified with the four usual modes, namely, a fast and a slow compression wave and two shear waves.

\section{Wave velocities}

The moduli of the wave velocities, for $\boldsymbol{p}$ set along $x, y$, and $z$, are plotted as functions of the frequency in Figs. 2, 3, and 4.

The fast compression wave velocity (Fig. 2) in the saturated medium is slower than in the dry medium, because of the additional inertia due to the interstitial fluid. It increases with the frequency. This effect becomes perceptible for $\omega_{c} \geqslant 10$, i.e., when $F$ exceeds a few percent, in agreement with result (84). The propagation along $y$ is faster than along $x$ and $z$. This is consistent with anisotropy of $\boldsymbol{D}_{\{4\}}$ in (A1), where $D_{22}>D_{11}, D_{33}$.

The shear wave velocities (Fig. 3) are also smaller in the saturated than in the dry medium because of the additional

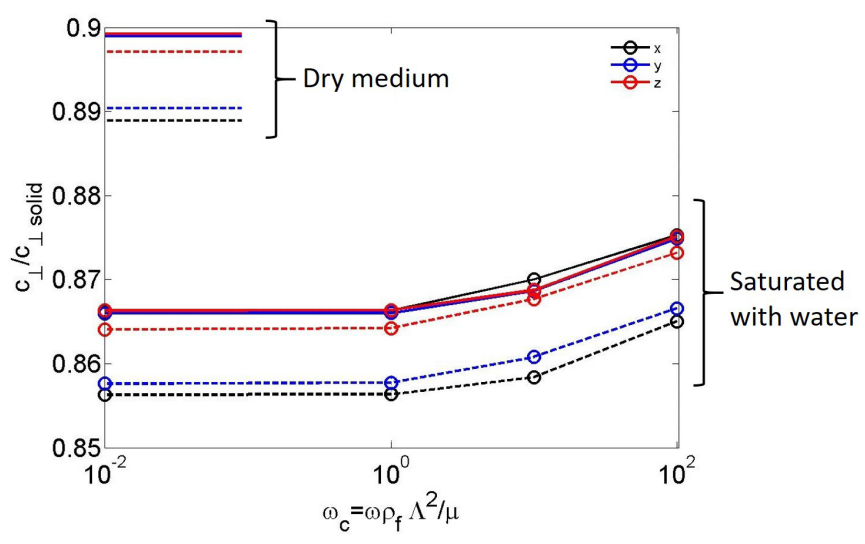

FIG. 3. The velocities of the two shear waves, normalized by the velocity in the solid as a function of the dimensionless frequency $\omega_{c}$ calculated along the directions $x, y$, and $z$. 


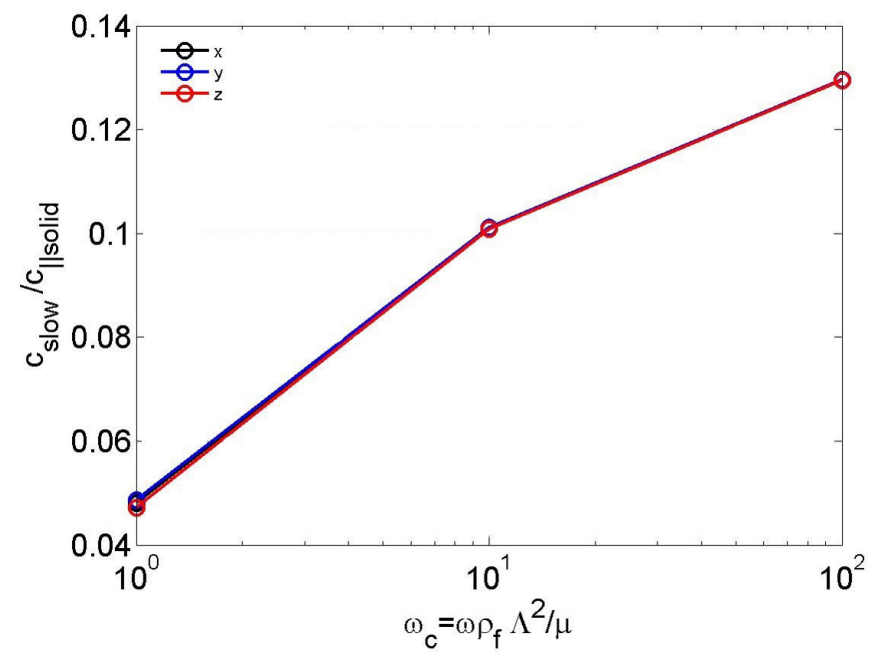

FIG. 4. The slow compression velocity, normalized by the velocity in the solid, as a function of the dimensionless frequency $\omega_{c}$ calculated along the directions $x, y$, and $z$.

fluid mass. Again, $c_{\perp}$ starts to increase with the frequency when $F$ exceeds a few percent, in agreement with (78). The velocities for the two shear waves are equal when $\boldsymbol{p}$ is parallel to $z$, but they differ for $\boldsymbol{p}$ along $x$ or $y$. This suggests a better isotropy around the $z$ axis than around the $x$ and $y$ axes. This can be connected to the properties of the lower-right block of $\boldsymbol{D}_{\{4\}}$ in (A1). It has an eigenvector nearly along $z$, with eigenvalue 0.251 , and two eigenvectors in the $x y$ plane, with the larger and almost-equal eigenvalues 0.256 and 0.258 .

Finally, the velocity of the slow wave is shown in Fig. 4. It is found to be nearly identical along the $x, y$, and $z$ directions. This seems surprising since $c_{\text {slow }}$ is proportional to the permeability according to (86); $\boldsymbol{K}$ is very anisotropic. This is because the principal directions of $\boldsymbol{K}$ are along diagonal directions, and its anisotropy is not perceived when operating along the axes, just as the anisotropy of the eigenvalues in (B2) does not show up when considering the diagonal terms in (B1). The increase in $c_{\text {slow }}$ with the frequency is in fair agreement with (86), which predicts $c_{\text {slow }} / c_{\| \text {solid }} \simeq 0.06,0.15$, and 0.21 when $\omega_{c}=1,10$, and 100 , respectively.

\section{CONCLUDING REMARKS}

The homogenization approach has been applied to study wave propagation through saturated porous media. Two

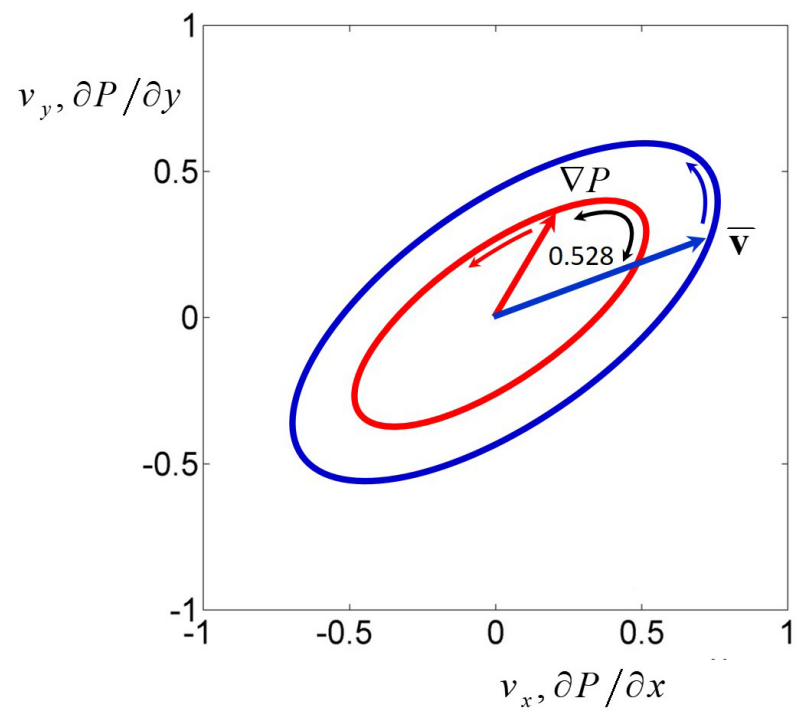

FIG. 5. Sketch of the evolutions of the pressure gradient $\nabla P$ and of the mean velocity $\overline{\boldsymbol{V}}$ during a period when the eigenvalue and eigenvector of $\boldsymbol{K}$ are complex. $\nabla P$ and $\overline{\boldsymbol{V}}$ describe similar ellipses with a phase delay for $\overline{\boldsymbol{V}}$.

significant extensions have been made. First, the compressibility of the interstitial fluid has been taken into consideration, i.e., the pore space can be saturated with a Newtonian compressible fluid. Second, the pore space may be composed of several connected components which may or not percolate.

All the physical quantities involved in the macroscopic description are obtained by solving the corresponding local equations separately. The physical meanings of these quantities are given and the properties associated with these quantities are discussed. The interactions between the pore are examined and some strategies are developed to reduce the computational effort.

The global wave properties, namely, the velocity and attenuation coefficients, were derived by solving the Christoffel equation. Three types of waves were observed during propagation through the saturated media. The wave velocities were expressed explicitly, which allows analysis of the effect of various parameters.

A systematic application to a synthetic porous medium has been made in order to illustrate the methodology and its results.

\section{APPENDIX A: EFFECTIVE ELASTIC PROPERTIES}

$$
\begin{aligned}
& \text { 1. } D_{\{4\}} \text { and } \alpha^{i} \\
& \boldsymbol{D}_{\{4\}}=E_{S}\left(\begin{array}{l}
\mathbf{0 . 8 0 5 8 5 4} \\
\mathbf{0 . 3 0 0 6 2 3} \\
\mathbf{0 . 2 9 9 3 6 6} \\
0.004146 \\
0.003591 \\
0.005746
\end{array}\right. \\
& \left.\begin{array}{cc}
0.003591 & -0.005745 \\
-0.000575 & -0.006153 \\
0.000696 & -0.002525 \\
-0.000591 & 0.000855 \\
\mathbf{0 . 2 5 6 1 2 6} & -0.002158 \\
-0.002158 & \mathbf{0 . 2 5 2 1 6 0}
\end{array}\right)
\end{aligned}
$$

$\mathbf{0 . 2 9 9 3 6 1}$
$\mathbf{0 . 3 0 2 4 4 4}$
$\mathbf{0 . 8 0 0 0 2 5}$
-0.009481
0.000698
-0.002527




$$
\begin{aligned}
\boldsymbol{\alpha}^{1} & =\left(\begin{array}{ccc}
-0.408627 & -0.004659 & 0.000351 \\
-0.004659 & -0.398951 & -0.006450 \\
0.000351 & -0.006450 & -0.412407
\end{array}\right), \\
\boldsymbol{\alpha}^{2} & =\left(\begin{array}{ccc}
-0.023603 & -0.001112 & 0.001238 \\
-0.001112 & -0.023297 & -0.002401 \\
0.001238 & -0.002401 & -0.021653
\end{array}\right), \\
\boldsymbol{\alpha}^{3} & =\left(\begin{array}{ccc}
-0.002985 & 0.000116 & 0.000024 \\
0.000116 & -0.002664 & -0.000090 \\
0.000024 & -0.000090 & -0.002458
\end{array}\right), \\
\boldsymbol{\alpha}^{4} & =\left(\begin{array}{lll}
-0.001812 & -0.000110 & -0.000209 \\
-0.000110 & -0.001445 & -0.000050 \\
-0.000209 & -0.000050 & -0.001976
\end{array}\right), \\
\boldsymbol{\alpha}^{5} & =\left(\begin{array}{ccc}
-0.000615 & -0.000007 & 0.000082 \\
-0.000007 & -0.000697 & -0.000036 \\
0.000082 & -0.000036 & -0.000750
\end{array}\right) .
\end{aligned}
$$

\section{Matrix $\overline{\bar{B}}$}

$$
\overline{\bar{B}}=\left(\begin{array}{ccccc}
\mathbf{- 0 . 3 0 9 8 1 1 0 1} & \mathbf{0 . 0 1 1 9 7 9 4 3} & \mathbf{0 . 0 0 1 2 0 0 1 5} & \mathbf{0 . 0 0 0 7 3 2 8 8} & \mathbf{0 . 0 0 0 2 8 0 9 6} \\
\mathbf{0 . 0 1 1 9 7 8 6 8} & -\mathbf{0 . 0 2 6 5 4 2 5 3} & 0.00008993 & 0.00003698 & 0.00002426 \\
\mathbf{0 . 0 0 1 1 9 9 7 4} & 0.00008995 & -\mathbf{0 . 0 0 3 0 0 0 0 6} & 0.00000686 & 0.00000169 \\
\mathbf{0 . 0 0 0 7 3 3 0 6} & 0.00003700 & 0.00000686 & -\mathbf{0 . 0 0 1 9 3 9 1 8} & 0.00000222 \\
\mathbf{0 . 0 0 0 2 8 1 2 6} & 0.00002428 & 0.00000169 & 0.00000223 & -\mathbf{0 . 0 0 0 7 5 4 5 7}
\end{array}\right)
$$

\section{APPENDIX B: DYNAMIC PERMEABILITY}

The four numerical values of the dynamic tensor $\boldsymbol{K}$ are

$$
\begin{array}{ll}
\omega_{c}=0, & \frac{10^{3}}{a^{2}} \boldsymbol{K}=\left(\begin{array}{lll}
52.2 & 16.3 & -16.4 \\
16.3 & 53.7 & -17.8 \\
-16.3 & -18.0 & 49.9
\end{array}\right) ; \\
\omega_{c}=1, & \frac{10^{3}}{a^{2}} \boldsymbol{K}=\left(\begin{array}{ccc}
51.1 & 15.6 & -15.7 \\
15.6 & 52.6 & -17.2 \\
-15.7 & -17.3 & 49.1
\end{array}\right)+i\left(\begin{array}{ccc}
-6.2 & -3.4 & 3.4 \\
3.4 & -6.7 & 3.8 \\
3.4 & 3.8 & -5.6
\end{array}\right) ; \\
\omega_{c}=10, & \frac{10^{3}}{a^{2}} \boldsymbol{K}=\left(\begin{array}{ccc}
25.6 & 1.5 & -1.5 \\
1.5 & 25.4 & -1.6 \\
-1.5 & -1.6 & 25.6
\end{array}\right)+i\left(\begin{array}{ccc}
-21.5 & -7.5 & 7.5 \\
-7.5 & -22.0 & 8.2 \\
7.5 & 8.2 & -20.7
\end{array}\right) ; \\
\omega_{c}=100, & \frac{10^{3}}{a^{2}} \boldsymbol{K}=\left(\begin{array}{ccc}
1.44 & -.25 & 0.25 \\
0.25 & 1.44 & 0.27 \\
0.25 & 0.27 & 1.46
\end{array}\right)+i\left(\begin{array}{ccc}
-6.41 & -0.11 & 0.11 \\
-0.11 & -6.41 & 0.12 \\
0.11 & 0.12 & -6.40
\end{array}\right) .
\end{array}
$$

These results can be commented on as follows. The static permeability tensor for $\omega_{c}=0$ is anisotropic. Its eigenvalues are

$$
K=0.0858, \quad 0.0367, \quad 0.0333
$$

along the eigendirections

$$
\left(\begin{array}{c}
-0.5665 \\
-0.6029 \\
0.5617
\end{array}\right), \quad\left(\begin{array}{c}
0.7790 \\
-0.6144 \\
0.1248
\end{array}\right), \quad\left(\begin{array}{l}
0.2821 \\
0.4987 \\
0.8196
\end{array}\right) .
$$

When the pressure gradient $\nabla P$ is set along one of these directions, the flow is parallel to it and in phase with it.

At a low frequency, $\omega_{c}=1, K$ contains an imaginary part, but the changes with respect to the static case are small. The eigenvalues and eigenvectors are almost identical to (B2) and (B3), although they contain a small imaginary part. 
At a high frequency, $\omega_{c}=100, \boldsymbol{K}$ is mostly imaginary. The eigenvalues are almost identical and pure imaginary numbers:

$$
K=0.0009-0.0066 i, \quad 0.0017-0.0063 i, \quad 0.0017-0.0063 i .
$$

The associated eigenvectors have almost purely real coordinates:

$$
\left(\begin{array}{c}
0.5716+0.0028 i \\
0.5889 \\
-0.5714-0.0024 i
\end{array}\right), \quad\left(\begin{array}{c}
0.1492-0.0365 i \\
0.6098+0.0382 i \\
0.7766
\end{array}\right), \quad\left(\begin{array}{c}
0.8114 \\
-0.5049+0.0382 i \\
0.2890+0.0417 i
\end{array}\right) \text {. }
$$

When the pressure gradient is set along one of these directions, the flow is parallel to it and in quadrature with it. The eigenvectors, (B5), are not exactly aligned with those in (B3) in the static case, but they deviate from them by only about $1^{0}, 11^{0}$, and $12^{0}$.

The case of the intermediate frequency, $\omega_{c}=10$, is more complex. The real and imaginary parts of $\boldsymbol{K}$ are of similar orders of magnitude. The same applies to the eigenvalues

$$
K=0.0286-0.0369 i, \quad 0.0240-0.0143 i, \quad 0.0240-0.0130 i
$$

and to the associated eigenvectors

$$
\left(\begin{array}{c}
0.5681+0.0092 i \\
0.5953 \\
-0.5680-0.0107 i
\end{array}\right), \quad\left(\begin{array}{c}
0.7866 \\
-0.5856+0.0584 i \\
0.1732+0.0697 i
\end{array}\right), \quad\left(\begin{array}{c}
0.2445-0.0485 i \\
0.5346+0.0577 i \\
0.8054
\end{array}\right)
$$

The real parts of the eigenvectors are again close to those in the static case, with deviations of about $10^{0}, 6^{0}$, and $5^{0}$. However, their imaginary part is quite significant, and the physical meaning of this feature may deserve an explanation.

Consider, for instance, the eigenvalue

$$
K=0.024-0.014 i=0.0278 e^{-0.528 i}
$$

which is associated with the vector

$$
\left(\begin{array}{c}
0.79 \\
-0.59+0.06 i \\
0.17+0.07 i
\end{array}\right)=\left(\begin{array}{c}
0.79 \\
0.593 e^{3.04 i} \\
0.184 e^{0.391 i}
\end{array}\right)
$$

During a period, the pressure gradient $\frac{\partial P}{\partial x}$ oscillates between -0.79 and +0.79 . Meanwhile, $\frac{\partial P}{\partial y}$ oscillates between -0.593 and +0.593 , with a phase shift of 3.04 (similarly for $\frac{\partial P}{\partial z}$, with a phase shift of 0.391 ). Hence, during a period, the vector $\nabla P$ rotates and describes an ellipse as illustrated in Fig. 5. The flow describes the same ellipse dilated by a factor $-|\boldsymbol{K}| / \mu_{f}$ with a phase delay of -0.528 [see (B8)].

Such behavior is perfectly normal from a mathematical point of view, and it can always be expected in the range of intermediate frequencies, when the real and imaginary parts of the permeability tensor are of comparable orders of magnitude.

\section{APPENDIX C: SOLUTION OF THE CHRISTOFFEL EQUATION FOR $\omega_{c}=10$}

For $\boldsymbol{p}$ along the $x$ axis, the eigenvalue problem has four solutions:

$$
\begin{aligned}
& \sqrt{\frac{\rho_{s}}{E_{s}}} c=0.9709+0.0026 i, \quad \hat{\boldsymbol{U}}=\left(\begin{array}{c}
-0.9985+0.0015 i \\
0.0064-0.0006 i \\
-0.0032+0.0006 i
\end{array}\right), \\
& \hat{\boldsymbol{W}}^{1}=\left(\begin{array}{c}
0.0131+0.0159 i \\
0.0044+0.0009 i \\
-0.0045-0.0009 i
\end{array}\right) ; \\
& \sqrt{\frac{\rho_{s}}{E_{s}}} c=0.5395+0.0023 i, \quad \hat{\boldsymbol{U}}=\left(\begin{array}{c}
-0.0094-0.0009 i \\
-0.4772-0.0574 i \\
0.9133+0.0866 i
\end{array}\right), \\
& \hat{\boldsymbol{W}}^{1}=\left(\begin{array}{c}
0.0079+0.0027 i \\
0.0126+0.0119 i \\
-0.0158-0.0202 i
\end{array}\right) ;
\end{aligned}
$$

$$
\begin{aligned}
\sqrt{\frac{\rho_{s}}{E_{s}}} c=0.5323+0.0021 i, \quad \hat{\boldsymbol{U}}=\left(\begin{array}{l}
0.0023-0.3483 i \\
0.4145-0.5854 i \\
0.2237-0.3000 i
\end{array}\right) \\
\hat{\boldsymbol{W}}^{1}=\left(\begin{array}{c}
-0.0014+0.0014 i \\
-0.0016+0.0002 i \\
-0.0061-0.0028 i
\end{array}\right) \\
\sqrt{\frac{\rho_{s}}{E_{s}}} c=0.0963+0.0450 i, \quad \hat{\boldsymbol{U}}=\left(\begin{array}{c}
0.2843-0.2102 i \\
0.0023-0.0059 i \\
0.0108-0.0026 i
\end{array}\right) \\
\hat{\boldsymbol{W}}^{1}=\left(\begin{array}{c}
-3.643+2.480 i \\
-0.302+0.963 i \\
0.300-0.968 i
\end{array}\right)
\end{aligned}
$$

In the fast solution, $\hat{\boldsymbol{U}}$ is nearly purely real and aligned with $\boldsymbol{p}$. This can be regarded as an almost-pure compression wave. We denote the velocity $c_{\|}$. It is mostly real, meaning that the attenuation is weak. The fluid displacement $\hat{W}^{1}$ is small compared to the solid displacement $\hat{\boldsymbol{U}}$.

In the second and third solutions, $\hat{\boldsymbol{U}}$ is complex. However, its $y$ and $z$ components have almost-identical phases, which 


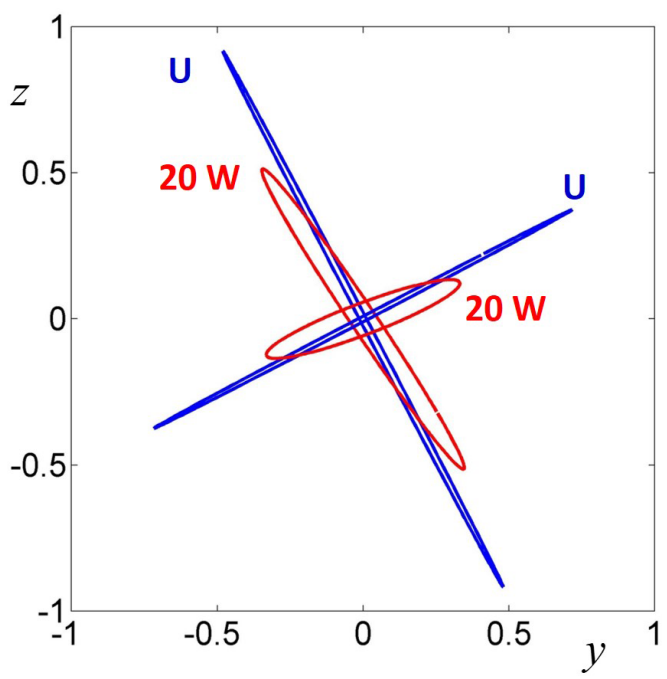

FIG. 6. Solid $(\hat{\boldsymbol{U}})$ and fluid $\left(\hat{\boldsymbol{W}}^{1}\right)$ displacements during a period, projected onto the $(y, z)$ plane orthogonal to $\boldsymbol{p}$, for the two shear waves with $\omega_{c}=10$. means that $\boldsymbol{U}$ oscillates along a single direction which is nearly othogonal to $\boldsymbol{p}$. Hence, these waves are predominantly shear waves. We denote the velocity $c_{\perp}$. Its imaginary part is comparatively larger than for $c_{\|}$, which means a stronger attenuation. However, the phases of the three components of the fluid displacement of $\hat{W}^{1}$ are not identical. This means that during a period $\hat{W}^{1}$ rotates around $\boldsymbol{p}$, as illustrated in Fig. 6. The induced flow $\hat{\boldsymbol{W}}^{1}$ is not exactly aligned with the solid shear $\hat{\boldsymbol{U}}$. Note that the two solutions for $\hat{U}$ and $\hat{W}^{1}$ do not appear orthogonal, especially for $\hat{W}^{1}$, because the $x$ component is not represented.

In the last solution, both the solid and the fluid displacements are aligned nearly along $\boldsymbol{p}$. The fluid displacement $\hat{\boldsymbol{W}}^{1}$ is much larger than the solid displacement $\hat{U}$. The velocity is much smaller than $c_{\|}$and the wave is strongly attenuated (large imaginary part). This is a slow compression wave which propagates mostly through the fluid. We denote the velocity $c_{\text {slow }}$.
[1] M. A. Biot, J. Acoust. Soc. Am. 28, 168 (1956).

[2] J. L. Auriault and E. Sanchez-Palencia, J. Mécanique 16, 575 (1977).

[3] J. L. Auriault, Int. J. Eng. Sci. 18, 775 (1980).

[4] R. Burridge and J. B. Keller, J. Acoust. Soc. Am. 70, 1140 (1981).

[5] C. Boutin and J. L. Auriault, Int. J. Eng. Sci. 28, 1157 (1990).

[6] C. Boutin and J. L. Auriault, Int. J. Eng. Sci. 31, 1669 (1993).

[7] I. Malinouskaya, V. V. Mourzenko, J.-F. Thovert, and P. M. Adler, Phys. Rev. E 77, 066302 (2008).

[8] S. R. Pride and J. G. Berryman, Phys. Rev. E 68, 036603 (2003).

[9] J. L. Auriault, Transp. Porous Media 64, 247 (2006).

[10] E. H. Saenger, F. Enzmann, Y. Keehm, and H. Steeb, J. Appl. Geophys. 74, 236 (2011).

[11] E. H. Saenger, D. Uribe, R. Janicke, O. Ruiz, and H. Steeb, Int. J. Eng. Sci. 58, 115 (2012).

[12] C. Perrot, F. Chevillotte, M. Tan Hoang, G. Bonnet, F.-X. Bécot, L. Gautron, and A. Duval, J. Appl. Phys. 111, 014911 (2012).

[13] T.-K. Nguyen, V. Monchiet, and G. Bonnet, Eur. J. Mech. B/Fluids 37, 90 (2013).
[14] M. T. Hoang, G. Bonnet, H. T. Luu, and C. Perrot, J. Acoust. Soc. Am. 135, 3172 (2014).

[15] T. G. Zieliński, J. Sound Vib. 409, 112 (2017).

[16] EPAPS Document No. E-PLEEE8-77-016806, Supplemental Material to [7]. Available at: http://ftp.aip.org/epaps/phys_rev_ e/E-PLEEE8-77-016806/.

[17] T. Lévy, Int. J. Eng. Sci. 17, 1005 (1979).

[18] I. N. Sneddon and N. F. Mott, Proc. R. Soc. London A 187, 229 (1997).

[19] V. Grechka, I. Vasconcelos, and M. Kachanov, Geophysics 71, D153 (2006).

[20] R. J. O'Connel and B. Budiansky, J. Geophys. Res. 79, 5412 (1974).

[21] P. M. Adler, C. G. Jacquin, and J. A. Quiblier, Int. J. Multiphase Flow 16, 691 (1990).

[22] D. L. Johnson, J. Koplik, and L. M. Schwartz, Phys. Rev. Lett. 57, 2564 (1986).

[23] A. Valfouskaya, P. M. Adler, J.-F. Thovert, and M. Fleury, J. Appl. Phys. 97, 083510 (2005).

[24] A. Pazdniakou and P. M. Adler, Adv. Water Resources 62, 292 (2013). 